Centre interuniversitaire de recherche en économie quantitative

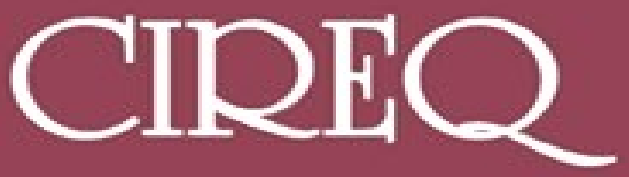

Cahier 17-2006

SOLIDARITY IN CHOOSING A LOCATION ON A CYCLE

Sidartha GORDON 


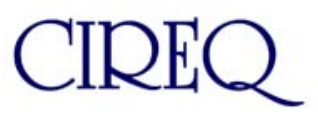

Le Centre interuniversitaire de recherche en économie quantitative (CIREQ) regroupe des chercheurs dans les domaines de l'économétrie, la théorie de la décision, la macroéconomie et marchés financiers, la microéconomie appliquée et économie expérimentale et l'économie de l'environnement et des ressources naturelles. Ils proviennent principalement des universités de Montréal, McGill et Concordia. Le CIREQ offre un milieu dynamique de recherche en économie quantitative grâce au grand nombre d'activités qu'il organise (séminaires, ateliers, colloques) et de collaborateurs qu'il reçoit chaque année.

The Center for Interuniversity Research in Quantitative Economics (CIREQ) regroups researchers in the fields of econometrics, decision theory, macroeconomics and financial markets, applied microeconomics and experimental economics, and environmental and natural resources economics. They come mainly from the Université de Montréal, McGill University and Concordia University. CIREQ offers a dynamic environment of research in quantitative economics thanks to the large number of activities that it organizes (seminars, workshops, conferences) and to the visitors it receives every year.

Cahier 17-2006

\section{SOLIDARITY IN CHOOSING A LOCATION ON A CYCLE} Sidartha GORDON

CIREQ, Université de Montréal C.P. 6128 , succursale Centre-ville Montréal (Québec) H3C 3J7 Canada téléphone : (514) 343-6557 télécopieur : (514) 343-5831 cireq@umontreal.ca http://www.cireq.umontreal.ca
Université th de Montréal
McGill

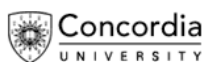


Ce cahier a également été publié par le Département de sciences économiques de l'Université de Montréal sous le numéro (2006-17).

This working paper was also published by the Department of Economics of the University of Montreal under number (2006-17).

Dépôt légal - Bibliothèque nationale du Canada, 2006, ISSN 0821-4441

Dépôt légal - Bibliothèque et Archives nationales du Québec, 2006

ISBN-13 : 978-2-89382-539-7; ISBN-10 : 2-89382-539-7 


\title{
Solidarity in Choosing a Location on a Cycle*
}

\author{
Sidartha Gordon ${ }^{\dagger}$ \\ March 2006, to appear in Social Choice and Welfare.
}

\begin{abstract}
We study the implications of two solidarity conditions on the efficient location of a public good on a cycle, when agents have single-peaked, symmetric preferences. Both conditions require that when circumstances change, the agents not responsible for the change should all be affected in the same direction: either they all gain or they all loose. The first condition, population-monotonicity, applies to arrival or departure of one agent. The second, replacement-domination, applies to changes in the preferences of one agent. Unfortunately, no Pareto-efficient solution satisfies any of these properties. However, if agents' preferred points are restricted to the vertices of a small regular polygon inscribed in the circle, solutions exist. We characterize them as a class of efficient priority rules.
\end{abstract}

Keywords: Location Theory; Cycle; Efficiency, Population-monotonicity, Replacement-Domination, Single-Peaked.

JEL classification: $\mathrm{C} 79, \mathrm{D} 71$

\footnotetext{
*Extensive comments and discussions with William Thomson are gratefully acknowledged. I am thankful for long discussions with Eiichi Miyagawa and James Schummer, and for comments by Rakesh Vohra, an anonymous referee, and audiences at Northwestern University and the Sixth International Meeting of the Society for Social Choice and Welfare, and for support from CIREQ.

†Université de Montréal, Départment de Sciences Économiques, C.P. 6128, succursale Centre-ville, Montréal QC H3C 3J7, Canada, E-mail: sidartha.gordon@umontreal.ca, Phone: (1)(514)343-2398, Fax: (514)343-7221.
} 


\section{Introduction}

We study the problem of choosing a location for a facility or a public good on a set of locations topologically equivalent to a cycle, on the basis of the preferences of agents over such locations. We assume that each agent has cyclically singlepeaked (and hence single-troughed) preferences. This means here that she has a preferred location; moving the good away from this location in either direction makes her worse off. Due to the structure of the cycle, she also has a worse location; moving the good away from this location in either direction makes her better off. We also assume that preferences are symmetric.

A spatial example of this problem is the choice of the location of a geostationary satellite above the equator. Each satellite user has a preferred location for the satellite, and the farther from this point the satellite is located, the worse off she is. Here is now a temporal example. Suppose that the government has to accomplish some task on a yearly basis, for example collecting taxes. Each inhabitant of the country has preferences over dates in the year for this to happen. Some prefer it to happen during the summer, while they are on vacation, others might prefer it to happen in January, when they are planning their budget for the year. Each has a preferred date and the closer the decided date is from his preferred date, the better off he is.

We wish to identify desirable location or date choice rules. First, it should not be possible to make all agents better off, and at least one of them strictly better off by replacing the chosen location or date by another one. This is the "Pareto-efficiency" requirement. Next, we consider independently two conditions of solidarity among agents when their circumstances change. These conditions ensure that changes affect everyone not responsible for these changes in the same direction. The first one applies to situations where the population of the economy is variable. Introduced by Thomson (1983a, 1983b) in the context of bargaining, "population-monotonicity" requires that when new agents 
arrive or when some agents leave, all the agents present at the beginning and at the end should be affected in the same direction. The second condition, "replacement-domination", applies to situations where the population of the economy is fixed. The changes in the environment are changes in preferences of the agents. It requires that if the preferences of one agent change, all the others should be affected in the same way: either all gain or all lose. Replacementdomination was introduced by Moulin (1987) in the context of binary public decision-making. Both conditions were extensively studied in bargaining theory, coalitional games, social choice theory and the theory of resource allocation. ${ }^{1}$

The axiomatic analysis of the choice of a location from a location set was first analyzed on a line. This study was later extended to more complex locations sets like trees, cycles or graphs containing at least one cycle. Pareto-efficiency is usually not restrictive in this class of problems. For example, when the location set is a line interval, over which agents have single-peaked preferences, the Pareto set is the entire segment of locations comprised between the two most extreme peaks. This motivated the study of Pareto-efficiency in conjunction with other axioms.

A first path was explored by Moulin (1980). He looked for strategy-proof rules, which give agents incentives to truthfully reveal their preferences. He considered the case where the location set is a line interval. Schummer and Vohra (2002) extended this work to the case of trees and graphs containing cycles. On cycles, Schummer and Vohra (2002) obtained a negative result: only dictatorial rules satisfy strategy-proofness and Pareto-efficiency.

A second approach, pioneered by Thomson (1993) and Ching and Thomson (1999) focuses on solidarity criteria. Like Moulin, they consider the case where the location set is a line interval. Thomson (1993) studied replacementdomination. Ching and Thomson (1999) studied population-monotonicity. Both

\footnotetext{
${ }^{1}$ For a survey on population-monotonicity, see Thomson (1999b). For a survey on replacement-domination, see Thomson (1999a).
} 
Thomson (1993) and Ching and Thomson (1999) characterized the following class of rules on the basis of Pareto-efficiency and solidarity. Let $a$ be a fixed point in the line interval. Whenever $a$ is located in the Pareto set, choose this point. Whenever it is not, choose the peak that is the closest to $a$. This defines a family of "target" rules parameterized by $a$. Vohra (1999) and Klaus (2001) extended these results to trees, and proved analogous characterizations in this case. The case of cycles (and graphs containing at least one cycle) was left open. $^{2}$

Unfortunately, when the location set is a cycle, no Pareto-efficient solution satisfies any of the two solidarity conditions, as we show in Section 3. The cyclic structure of the preferences and the richness of the domain render Pareto-efficiency and solidarity incompatible. Are these conditions compatible on a smaller domains of cyclically single-peaked preferences? We show that they are, when the domain is sufficiently small. In Section 4, we introduce a class of restricted finite domains of cyclically single-peaked preferences. Each of these domains is obtained by restricting agent's preferred points to lie on a regular polygon inscribed in the circle. We characterize the set of solutions on polygons with three, four and five vertices. Each of the solutions satisfying Paretoefficiency and solidarity has the following interesting feature: it maximizes a certain weak partial order on the Pareto-set. On the hexagon and any higher order regular polygon, we obtain an impossibility. Therefore, Pareto-efficiency and solidarity are compatible only on small domains of cyclically single-peaked preferences.

\footnotetext{
${ }^{2}$ Another ramification of this literature was initiated by Miyagawa $(1998,2001)$ and Ehlers $(2002,2003)$ who both considered the location of multiple goods on a line. In the location problem of two goods, they obtained different characterizations of Pareto-efficient and populationmonotonic rules corresponding to different preferences over pairs of locations. Ehlers and Klaus (2001) consider lotteries of locations on an interval. Gordon (2006) studies a model of "public decision", which generalizes all models of location choice, and derives general implications of solidarity conditions.
} 


\section{The Model}

The choice of the location of a facility on a location set topologically equivalent to a circle has to be made. In order to keep matters simple and with no loss of generality, let the location set ${ }^{3}$ be a circle $\mathcal{C}$ in the Euclidean space $\mathbb{R}^{2}$. Let $d(.,$. be the shortest distance along the circle. For all $x \in \mathcal{C}$, let $\sigma(x)$ be the location on $\mathcal{C}$ diametrally opposed to $x$, i.e. the location $y$ that maximizes $d(x, y)$.

Let $\mathcal{N}$ be the set of nonnegative integers. Each integer represents an agent. A population is a non-empty and finite subset $M \subset \mathcal{N}$. A preference $R$ on $\mathcal{C}$ is a binary relation, that is reflexive, transitive and complete. Let $\mathcal{R}$ be the set of all cyclically single-peaked and symmetric preferences. Each such preference $R \in \mathcal{R}$ has a preferred location $p(R)$. For all $x, y \in \mathcal{C}$, let $x R y$ iff $d(x, p(R)) \leq d(y, p(R))$. Therefore the worst location for preference $R$ is $\sigma(p(R))$. Let $P$ be the strict preference relation, and $I$ be the indifference relation associated with preference $R$.

Within a population $M$, each agent $i \in M$ is equipped with a preference relation $R_{i} \in \mathcal{R}$. A preference profile for population $M$ is a list $R_{M}=\left(R_{i}\right)_{i \in M}$. Let $\mathcal{R}^{M}$ be the set of preference profiles for population $M$. More generally, a preference profile is a list $R_{M}$ for some population $M$. It is therefore an element of the union of all $\mathcal{R}^{M}$, over all populations $M$. Let $\mathcal{U}$ be this union. For all two by two disjoint populations $M_{1}, \ldots, M_{k}$, the notation $\left(R_{M_{1}}, \ldots, R_{M_{k}}\right)$ designates the profile for the population $M_{1} \cup \ldots \cup M_{k}$, such that, for all $j=1, \ldots, k$, agents in $M_{j}$ have preferences as in the profile $R_{M_{j}}$.

For all $R_{M} \in \mathcal{U}$, all pair of locations $x, y \in \mathcal{C}$, the location $x$ weakly-Paretodominates $y$ for $R_{M}$ if, for all $i \in M$, we have $x R_{i} y$. This is denoted by $x$ $R_{M} y$. Similarly, $x$ is Pareto-indifferent to $y$ for $R_{M}$ if, for all $i \in M$, we have

\footnotetext{
${ }^{3}$ The specific geometric shape of the location set does not play any role. With a different geometric shape, the problem is homeomorphic to the one we consider and all the results apply.
} 
$x I_{i} y$. This is denoted by $x I_{M} y$. For all $R_{M} \in \mathcal{U}$, a location $x \in \mathcal{C}$ is Paretoefficient for $R_{M}$ if no other location in $\mathcal{C}$ weakly-Pareto-dominates $x$ and is strictly preferred by at least one agent in $M$, i.e. if there is no $y \in \mathcal{C}$ such that $y R_{M} x$ and $y P_{i} x$ for some $i \in M$. Let $E\left(R_{M}\right)$ be the set of Pareto-efficient locations for $R_{M}$. As we will argue later, this set if generally a continuum. This motivates the search for a selection from the Pareto-set that satisfies other desirable conditions.

A solution maps preference profiles from a certain subset $\mathcal{D}$ of $\mathcal{U}$ into $\mathcal{C}$. For all $R_{M} \in \mathcal{D}$, the location selected by the solution for the profile $R_{M}$ is interpreted as a prescription for the profile $R_{M}$. A solution on $\mathcal{D}$ is Pareto-efficient if for all $R_{M} \in \mathcal{D}$, it selects for $R_{M}$ a location in the Pareto-set $E\left(R_{M}\right)$. We are interested in Pareto-efficient solutions that satisfy in addition a solidarity condition. Such conditions are defined next.

\section{$2.1 \quad$ Population-monotonicity}

In the variable population model, a solution is denoted by $\Pi$ and its domain is $\mathcal{U}$. We search for Pareto-efficient solutions $\Pi: \mathcal{U} \rightarrow \mathcal{C}$ satisfying the solidarity requirement of population-monotonicity. This conditions says that when one agent joins a population, all other agents initially present (whose preferences are kept fixed), should be affected in the same direction. Solution $\Pi$ satisfies this condition if, for all $R_{M} \in \mathcal{U}$, all $i \in \mathcal{N} \backslash M$, and all $R_{i}^{\prime} \in \mathcal{R}$, we have, either $\Pi\left(R_{i}^{\prime}, R_{M}\right) R_{M} \Pi\left(R_{M}\right)$, or $\Pi\left(R_{M}\right) R_{M} \Pi\left(R_{i}^{\prime}, R_{M}\right)$. Together with Paretoefficiency, population-monotonicity implies ${ }^{4}$ a stronger property, populationmonotonicity $_{+}$. This conditions says that when a group of agents joins a population, all other agents initially present (whose preferences are kept fixed), should weakly lose. A solution satisfies this condition if, for all $R_{M}, R_{L}^{\prime} \in \mathcal{U}$,

\footnotetext{
${ }^{4}$ This result is standard in the literature. See for example Ehlers and Klaus (2001) or Gordon (2006).
} 
such that $L \cap M=\emptyset$, we have $\Pi\left(R_{M}\right) R_{M} \Pi\left(R_{L}^{\prime}, R_{M}\right)$.

\subsection{Replacement-domination}

In the fixed population model, let $N$ be the fixed population consisting of the $n \geq 3$ agents with labels $0, \ldots, n-1$. A solution is denoted by $\Phi$ and its domain is $\mathcal{R}^{N}$. We search for Pareto-efficient solutions $\Phi: \mathcal{R}^{N} \rightarrow \mathcal{C}$ satisfying the solidarity requirement of replacement-domination. This condition says that if the preferences of one agent change, all the other agents (whose preferences are kept fixed) should be affected in the same direction. Solution $\Phi$ satisfies this condition if, for all $i \in N$, for all $R_{N \backslash\{i\}} \in \mathcal{R}^{N \backslash\{i\}}$, and for all $R_{i}, R_{i}^{\prime} \in \mathcal{R}$, we have, either $\Phi\left(R_{i}^{\prime}, R_{N \backslash\{i\}}\right) R_{N \backslash\{i\}} \Phi\left(R_{i}, R_{N \backslash\{i\}}\right)$, or $\Phi\left(R_{i}, R_{N \backslash\{i\}}\right) R_{N \backslash\{i\}}$ $\Phi\left(R_{i}^{\prime}, R_{N \backslash\{i\}}\right)$.

\section{Full domain}

In this section, we describe the structure of the Pareto-set for any profile in $\mathcal{U}$, and present negative results. No Pareto-efficient solution on $\mathcal{U}$ satisfies population-monotonicity. No Pareto-efficient solution on $\mathcal{R}^{N}$ satisfies replacement-domination.

We first introduce the following definitions. An arch is a connected subset of $\mathcal{C}$. For all $x, y \in \mathcal{C}, x \neq y$, the closed arch $[x, y]$ is the set of locations including $x$ and $y$, that are reached as we travel counter-clockwise between $x$ and $y$. Define similarly (with the appropriate modifications) the open arch ]$x, y[$, and the semi-open arches $[x, y[$ and $] x, y]$. For all $x, y \in \mathcal{C}, x \neq y$, we have $[x, y] \cup] y, x[=[x, y[\cup[y, x[=] x, y] \cup] y, x]=\mathcal{C}$. By convention, let $[x, x]:=\{x\}$. For all $x \in \mathcal{C}$, let $R^{x} \in \mathcal{R}$ be the unique preference such that $p\left(R^{x}\right)=x$. For all $R_{M} \in \mathcal{U}$, let $S\left(R_{M}\right)$ be the set $\left\{R_{i}: i \in M\right\}$.

In order to characterize the Pareto-set, we introduce a taxonomy of profiles in $\mathcal{U}$. First, profiles in $\mathcal{L}$ (as in "large") are the ones with the highest preference 
heterogeneity. Let $R_{M} \in \mathcal{L}$ if it is not possible to find a single half-circle containing all peaks in the profile, i.e. there are $x, y, z$ such that $y \in] x, \sigma(x)[$, $z \in] \sigma(x), x\left[,\left\{R^{x}, R^{y}, R^{z}\right\} \subseteq S\left(R_{M}\right)\right.$, and either $R^{\sigma(x)} \in S\left(R_{M}\right)$ or $z \in$ ]$y, \sigma(y)[$. Second, profiles in $\mathcal{P}$ (as in "polar") are the ones with polarized preferences. Let $R_{M} \in \mathcal{P}$ if exactly two preferences are represented in the profile, one the opposite of the other, i.e. there is $x \in \mathcal{C}$ such that $S\left(R_{M}\right)=\left\{R^{x}, R^{\sigma(x)}\right\}$. Third, profiles in $\mathcal{I}$ (as in "interval") are the ones with the lowest heterogeneity of preferences. Let $R_{M} \in \mathcal{I}$ if there are $x, z \in \mathcal{C}$, such that $z \in[x, \sigma(x)]$, $R^{x}, R^{z} \in S\left(R_{M}\right) \subseteq\left\{R^{y}: y \in[x, z]\right\}$ and $S\left(R_{M}\right) \nsubseteq\left\{R^{x}, R^{\sigma(x)}\right\}$. For all such profile $R_{M}$, there is at most one such pair $(x, z)$. In particular, the class $\mathcal{I}$ contains the subclass of unanimous profiles, such that all agents in the profile share the same preference. Clearly, $\mathcal{L}, \mathcal{P}$ and $\mathcal{I}$ partition $\mathcal{U}$. The following result motivates the above profile taxonomy.

Lemma 1 For all $R_{M} \in \mathcal{L} \cup \mathcal{P}$, we have $E\left(R_{M}\right)=\mathcal{C}$. For all $R_{M} \in \mathcal{I}$, let $(x, z)$ be the unique pair of locations such that $z \in[x, \sigma(x)]$, and $R^{x}, R^{z} \in S\left(R_{M}\right) \subseteq$ $\left\{R^{y}: y \in[x, z]\right\}$. Then $E\left(R_{M}\right)=[x, z]$.

Proof. For profiles in $\mathcal{I}$, the result is simply analogous to the case where the location set is a line. It is therefore the interval delimited by the most extreme peaks in the profile, i.e. the interval $[x, z]$ where $x$ and $z$ are as in the definition above. For any profile in $\mathcal{P}$, all locations are Pareto-efficient since the two polar preferences in the profile are each the opposite of the other. For any profile $R_{M}$ in $\mathcal{L}$, and any location $x$ in $\mathcal{C}$, we can find a subpopulation $\{i, j\} \subseteq M$ such that the profile $\left(R_{i}, R_{j}\right)$ is an element of $\mathcal{I}$, and $x$ is an element of $E\left(R_{i}, R_{j}\right)=\left[p\left(R_{i}\right), p\left(R_{j}\right)\right]$. Then no other location even weakly Paretodominates $x$ for $\left(R_{i}, R_{j}\right)$. As a consequence, $x \in E\left(R_{M}\right)$. Therefore, $E\left(R_{M}\right)=\mathcal{C}$ in this case as well.

The Pareto set is therefore a continuum, except for unanimous profiles. 
This motivates the search for Pareto-efficient solutions satisfying solidarity conditions. Unfortunately, none exists, as we show next. This result contrasts with the positive results obtained by Thomson (1993) and Ching and Thomson (1999), when the location set is a line. The proof of this result is deferred to the end of the paper, since it is an implication of a stronger result (Theorem 6).

Theorem 1 No solution on $\mathcal{U}$ satisfies Pareto-efficiency and population-monotonicity.

Let $N \in \mathcal{N}$ such that $|N| \geqslant 4$. No solution on $\mathcal{R}^{N}$ satisfies Pareto-efficiency and replacement-domination.

The following lesson can be drawn from this disappointing result. Solidarity and Pareto-efficiency are incompatible in a sufficiently rich domain of cyclically singe-peaked preferences (in this case, when all such preferences are admissible). However, as we will show next, these axioms are compatible in smaller domains. How small must a domain be for Pareto-efficiency and solidarity to be compatible? Which solutions satisfy these conditions on small domains? Section 4 provides a detailed answer to these two questions.

We end this section by stating a useful property of the Pareto set in this model, which follows directly from the previous definitions. The Pareto set can always be generated by a small sub-profile.

Lemma 2 For all $R_{M} \in \mathcal{U}$, there exists a subpopulation $L \subseteq M$ such that $|L| \leq 4$ and $E\left(R_{L}\right)=E\left(R_{M}\right)$. When $R_{M} \in \mathcal{P} \cup \mathcal{I}$, this subpopulation can be chosen such that $|L| \leq 2$.

\section{Polygon-structured preferences}

We introduce a family $\mathcal{R}_{v}$ of discrete subsets of $\mathcal{R}$ indexed by $v \in \mathbb{N}$ such that $v \geq 3$. For each such $v$, let $c_{0}, \ldots, c_{v-1}$ (in counterclockwise order) be the $v$ vertices of a regular polygon inscribed in $\mathcal{C}$. Let $\mathbb{N}_{v}$ be the set $0, \ldots, v-1$. For 
convenience, for all $s \in \mathbb{Z}$, let $c_{s}:=c_{r}$, where $r$ is the unique element of $\mathbb{N}_{v}$ such that $s-r$ is divisible by $v$. Equivalently, $r$ is the remainder in the euclidian division of $s$ by $v$. For all $s \in \mathbb{Z}$, let $R^{s}$ be the unique preference in $\mathcal{R}$ such that $p\left(R^{s}\right)=c_{s}$. Let $\mathcal{R}_{v}$ be the set of preferences in $\mathcal{R}$ whose peaks are one of the $v$ vertices, i.e. $\mathcal{R}_{v}:=\left\{R^{0}, \ldots, R^{v-1}\right\}$. Let $\mathcal{U}_{v} \subset \mathcal{U}$ be the set of profiles in $\mathcal{U}$ such that all agents have preferences in $\mathcal{R}_{v}$. In this section, we show that, if $v$ is less or equal than five, then (i) Pareto-efficient solutions restricted to profiles in $\mathcal{U}_{v}$ satisfying population-monotonicity exist, and (ii) Pareto-efficient solutions restricted to profiles in $\mathcal{R}_{v}^{N}$ satisfying replacement-domination exist. We characterize these solutions. ${ }^{5}$

\subsection{The Pareto set}

In the particular case where $R_{M} \in \mathcal{R}_{v}$, the previous taxonomy can be simplified as follows. For all profile $R_{M}$, we have $R_{M} \in \mathcal{L}$ if there are $r, s, t \in \mathbb{N}_{v}$ such that $\left.c_{s} \in\right] c_{r}, \sigma\left(c_{r}\right)\left[, c_{t} \in\right] \sigma\left(c_{r}\right), c_{r}\left[\right.$, and $\left\{R^{r}, R^{s}, R^{t}\right\} \subseteq S\left(R_{M}\right)$, and either $(v$ is even and $\left.R^{r+\frac{v}{2}} \in S\left(R_{M}\right)\right)$ or $\left.c_{t} \in\right] c_{s}, \sigma\left(c_{s}\right)\left[\right.$. We have $R_{M} \in \mathcal{P}$ if $v$ is even, and there is $r \in \mathbb{N}_{v}$ such that $S\left(R_{M}\right)=\left\{R^{r}, R^{r+\frac{v}{2}}\right\}$. Finally, we have $R_{M} \in \mathcal{I}$ if there are $r, t \in \mathbb{N}_{v}$, such that $c_{t} \in\left[c_{r}, \sigma\left(c_{r}\right)\right]$, and $R^{r}, R^{t} \in S\left(R_{M}\right) \subseteq\left\{R^{s}: c_{s} \in\left[c_{r}, c_{t}\right]\right\}$ and $R_{M} \notin \mathcal{P}$. In this last case, the pair $(r, t)$ is uniquely determined, and from Lemma 2, we have $E\left(R_{M}\right)=\left[c_{r}, c_{t}\right]$.

\subsection{An implication of population-monotonicity}

In Lemma 3, we establish a general implication of Pareto-efficiency and populationmonotonicity on the discrete domains $\mathcal{U}_{v}$. If a solution satisfies populationmonotonicity and Pareto-efficiency, then its restriction to non-polar profiles only depends on the Pareto-set, and satisfies a monotonicity property with re-

\footnotetext{
${ }^{5}$ Many of the objects we introduce, such as the locations $c_{s}$ and the preferences $R^{s}$, depend on the parameter $v$. To alleviate notations, we do not index these objects by $v$.
} 
spect to the Pareto-set. Later, we will prove that for $v \in\{3,4,5\}$, solutions on $\mathcal{U}_{v}$ satisfying these conditions exist. For these cases, Lemma 3 describes a feature common to all such solutions. But we will also prove that, for $v>5$, no solution on $\mathcal{U}_{v}$ satisfies these conditions. For these cases, Lemma 3 is a step towards the impossibility result.

Lemma 3 Suppose that there exists a solution on $\mathcal{U}_{v}$ satisfying Pareto-efficiency and population-monotonicity, and let $\Pi$ be such a solution. Let $R_{N}, R_{M}^{\prime} \in \mathcal{U}_{v} \backslash \mathcal{P}$ such that $\Pi\left(R_{N}\right) \in E\left(R_{M}^{\prime}\right) \subseteq E\left(R_{N}\right)$. Then $\Pi\left(R_{M}^{\prime}\right)=\Pi\left(R_{N}\right)$.

Proof. Let $L$ be a population such that $L \cap M=L \cap N=\emptyset$, and let $R_{L}^{\prime \prime} \in$ $\mathcal{U}_{v} \backslash \mathcal{P}$ such that $S\left(R_{L}^{\prime \prime}\right)=S\left(R_{M}^{\prime}\right)$. An implication of this equality is that, for all $x, y \in \mathcal{C}$, the statements $x R_{L}^{\prime \prime} y$ and $x R_{M}^{\prime} y$ are equivalent. Therefore, $E\left(R_{L}^{\prime \prime}\right)=E\left(R_{M}^{\prime}\right) \subseteq E\left(R_{N}\right)$. Let $x_{N}:=\Pi\left(R_{N}\right), x_{M}:=\Pi\left(R_{M}^{\prime}\right), x_{L}:=\Pi\left(R_{L}^{\prime \prime}\right)$, $x_{M L}:=\Pi\left(R_{M}^{\prime}, R_{L}^{\prime \prime}\right)$ and $x_{N L}:=\Pi\left(R_{N}, R_{L}^{\prime \prime}\right)$. By population-monotonicity, , we have $x_{M} R_{M}^{\prime} x_{M L}$. Since $x_{M L} \in E\left(R_{M}^{\prime}, R_{L}^{\prime \prime}\right)=E\left(R_{M}^{\prime}\right)$, we have $x_{M} I_{M}^{\prime} x_{M L}$. Since $R_{M}^{\prime} \notin \mathcal{P}$, this implies in fact $x_{M}=x_{M L}$. By a similar argument, we have $x_{M L}=x_{L}$. Therefore $x_{M}=x_{L}$. By population-monotonicity ${ }_{+}$, we have $x_{N} R_{N}$ $x_{N L}$. Since $x_{N L} \in E\left(R_{N}, R_{L}^{\prime \prime}\right)=E\left(R_{N}\right)$, we have in fact $x_{N} I_{N} x_{N L}$. Since $R_{N} \notin \mathcal{P}$, this implies $x_{N}=x_{N L}$. By population-monotonicity ${ }_{+}$, we have $x_{L} R_{L}^{\prime \prime}$ $x_{N L}$. Since $x_{N L}=x_{N}$ and $x_{L}=x_{M}$, we then have $x_{M} R_{L}^{\prime \prime} x_{N}$. Recall that for all $x, y \in \mathcal{C}$, the statements $x R_{L}^{\prime \prime} y$ and $x R_{M}^{\prime} y$ are equivalent. Therefore $x_{M}$ $R_{M}^{\prime} x_{N}$. But since $x_{N} \in E\left(R_{M}^{\prime}\right)$, we then obtain $x_{M} I_{M}^{\prime} x_{N}$. Since $R_{M}^{\prime} \notin \mathcal{P}$, this implies $x_{M}=x_{N}$.

\subsection{Implications of replacement-domination}

In Lemma 4 and Theorem 2, we establish general implications of Pareto-efficiency and replacement-domination on the discrete domains $\mathcal{R}_{v}^{N}$. In Lemma 4, we show in essence that if a solution satisfies these conditions, then its restriction 
to non-polar profiles only depends on the Pareto-set. In Theorem 2, we show that replacement-domination is at least as strong a requirement as populationmonotonicity, in the sense that any solution that satisfies Pareto-efficiency and replacement-domination on $\mathcal{R}_{v}^{N}$ can be extended to a solution on $\mathcal{U}_{v}$ satisfying Pareto-efficiency and population-monotonicity. Later, we will prove that for $v \in\{3,4,5\}$, solutions on $\mathcal{R}_{v}^{N}$ satisfying Pareto-efficiency and replacementdomination exist. For these cases, Lemma 4 and Theorem 2 describe common features of all such solutions. But we will also prove that for $v>5$, no solution on $\mathcal{R}_{v}^{N}$ satisfies these conditions. For these cases, Lemma 4 and Theorem 2 are steps towards the impossibility result.

Recall that $N$ is a fixed population, such that $|N| \geq 4$. In Lemma 4, we use the following notation. For all $r, s \in \mathbb{Z}$, let $R_{N}^{r * s}:=\left(R^{r}, R^{s}, \ldots, R^{s}\right)$. Also let $x_{r * s}:=\Phi\left(R_{N}^{r * s}\right)$. For all $s \in \mathbb{Z}$ and all $x \in \mathcal{C}$, let $\sigma_{s}(x):=x$ if $x=c_{s}$, and let $\sigma_{s}(x)$ be the unique location in $\mathcal{C}$ distinct from $x$ that is indifferent to $x$ for preference $R^{s}$.

Lemma 4 Suppose that there exists a solution on $\mathcal{R}_{v}^{N}$ that satisfies Paretoefficiency and replacement-domination, and let $\Phi$ be such a solution. ( $i$ ) For all $R_{N} \in \mathcal{I}$, let $(r, s)$ be the unique indices such that $E\left(R_{N}\right)=\left[c_{r}, c_{s}\right]$. If $c_{s} \neq \sigma\left(c_{r}\right)$, we have $\Phi\left(R_{N}\right)=x_{r * s}$. (ii) There exists $x_{\Phi}^{*} \in \mathcal{C}$ such that, for all $R_{N} \in \mathcal{L}$, we have $\Phi\left(R_{N}\right)=x_{\Phi}^{*}$, for all $R_{N} \in \mathcal{P}$, we have $\Phi\left(R_{N}\right) I_{N} x_{\Phi}^{*}$, and for all $s \in \mathbb{Z}$, and for all profile $R_{N} \in \mathcal{R}_{v}^{N}$ such that $E\left(R_{N}\right)=\left[c_{s}, \sigma\left(c_{s}\right)\right]$, we have $\Phi\left(R_{N}\right)=x_{\Phi}^{*}$ if $x_{\Phi}^{*} \in E\left(R_{N}\right)$ and $\Phi\left(R_{N}\right)=\sigma_{s}\left(x_{\Phi}^{*}\right)$ otherwise.

Proof. We will prove $(i)$ and $(i i)$, after two preliminary steps.

Step 0 (reduction): Let $R_{N} \in \mathcal{R}_{v}^{N}$. Let $L \subset N$ be nonempty. Suppose that for all $M$ such that $L \subseteq M \subseteq N$, we have $E\left(R_{M}\right) \subseteq E\left(R_{L}\right)$. Let $R_{N}^{\prime}$ be such that $R_{L}^{\prime}=R_{L}$ and $S\left(R_{N}^{\prime}\right)=S\left(R_{L}\right)$. Then $\Phi\left(R_{N}\right) I_{L} \Phi\left(R_{N}^{\prime}\right)$. If, in addition, $R_{N}^{\prime} \notin \mathcal{P}$, then $\Phi\left(R_{N}\right)=\Phi\left(R_{N}^{\prime}\right)$. 
Proof of Step 0: Let $i(1), \ldots, i(K)$ denote the agents in $N \backslash L$. Consider the sequence of profiles such that $R_{N}^{0}:=R_{N}$ and profile $R_{N}^{k}$ is identical to profile $R_{N}^{k-1}$, except for agent $i(k)$, whose preference is replaced with $R_{i(k)}^{\prime}$, so that $R_{N}^{k}:=\left(R_{N \backslash\{i(k)\}}^{k-1}, R_{i(k)}^{\prime}\right)$. Clearly $R_{N}^{K}=R_{N}^{\prime}$. Along the transformation path, the preferences of agents in $L$ remain fixed at $R_{L}$. We have $\Phi\left(R_{N}^{k}\right) \in E\left(R_{N}^{k}\right) \subseteq$ $E\left(R_{L}\right)$, by Pareto-efficiency of $\Phi$ and the assumption on the Pareto-sets. This and replacement-domination for the fixed subprofile $R_{L}$ imply that the entire sequence $\left\{\Phi\left(R_{N}^{k}\right)\right\}_{0}^{n-3}$ lies in a single indifference curve for each preference in the profile $R_{L}$. Therefore $\Phi\left(R_{N}\right) I_{L} \Phi\left(R_{N}^{\prime}\right)$. If, in addition, $R_{N}^{\prime} \notin \mathcal{P}$, we obtain further $\Phi\left(R_{N}\right)=\Phi\left(R_{N}^{\prime}\right)$.

Step 1: Suppose that $R^{r}, R^{s} \in S\left(R_{N}\right)$ and for all $M \subseteq N$, we have $E\left(R_{M}\right) \subseteq$ $E\left(R^{r}, R^{s}\right)$. Then $\Phi\left(R_{N}\right) I_{N}^{r * s} x_{r * s}$.

Proof of Step 1: The result is trivially satisfied if $r=s$, so let us assume $r \neq s$. Let $i$ such that $R_{i}=R^{r}$, and $j$ such that $R_{j}=R^{s}$. Let $l \neq i, j$. Let $R_{l}^{\prime}:=R_{1}$. Let $R_{N}^{\prime}:=\left(R_{N \backslash\{l\}}, R_{l}^{\prime}\right)$. Let $R_{N}^{\prime \prime}:=\left(R_{N \backslash\{l\}}^{\prime}, R^{r}\right)$. By replacement-domination, applied to agents $i$ and $j$, we have $\Phi\left(R_{N}^{\prime}\right) I_{N}^{r * s} \Phi\left(R_{N}\right)$. By replacement-domination, applied to agents in $\{i, j, l\} \backslash\{1\}$, we have $\Phi\left(R_{N}^{\prime \prime}\right) I_{N}^{r * s} \Phi\left(R_{N}^{\prime}\right)$. If $j \neq 1$, let $g:=j$, otherwise let $g:=l$. In both cases $g \neq 1$ and $R_{g}^{\prime \prime}=R^{s}$. By Step 0 applied to $L:=\{1, g\}$ and $R^{\prime}:=R_{N}^{r * s}$, we have $\Phi\left(R_{N}^{\prime \prime}\right) I_{N}^{r * s} x_{r * s}$.

Proof of $(i)$. Apply Step 1 in the special case, where $c_{s} \neq \sigma\left(c_{r}\right)$. The result is trivially satisfied if $r=s$, so let us assume $r \neq s$. In this case, $\Phi\left(R_{N}^{\prime \prime}\right) I_{N}^{r * s} x_{r * s}$ implies $\Phi\left(R_{N}^{\prime \prime}\right)=x_{r * s}$.

Proof of $(i i)$. We distinguish two cases, depending on whether $v$ is odd or even.

Case 1. $v \geq 3$ is odd. In this case, there are no profiles in $\mathcal{P}$, so it suffices to prove the claim for profiles in $\mathcal{L}$. Let $\eta:=\frac{v-1}{2}$. Let $x_{\Phi}^{*}:=\Phi\left(R^{0}, R^{\eta}, R^{\eta+1}, \ldots, R^{\eta+1}\right)$. Let $R_{N} \in \mathcal{L}$. We will show that $\Phi\left(R_{N}\right)=x_{\Phi}^{*}$. We now define a sequence $\left\{R_{N}^{* k}\right\}_{k=0, \ldots, 6}$ of profiles, a sequence $\{i(k)\}_{k=1, \ldots, 6}$ of agents, and a sequence 
$\left\{\underline{R}_{i(k)}^{* k}\right\}_{k=1, \ldots, 6}$ of "replacement preferences". Let $R_{N}^{* 0}:=R_{N}$ and or all $k=$ $1, \ldots, n$, let $R_{N}^{* k}$ be the profile obtained from $R_{N}^{* k-1}$ by replacing the preference of agent $i(k)$ with the preference $\underline{R}_{i(k)}^{* k}$, so that $R_{N}^{* k}:=\left(R_{N \backslash\{i(k)\}}^{* k-1}, \underline{R}_{i(k)}^{* k}\right)$. Let $\underline{R}_{i(1)}^{* 1}:=R_{1}^{* 0}, \underline{R}_{i(2)}^{* 2}:=R^{0}, \underline{R}_{i(3)}^{* 3}:=R_{2}^{* 2}, \underline{R}_{i(4)}^{* 4}:=R^{\eta}, \underline{R}_{i(5)}^{* 5}:=R_{3}^{* 4}, \underline{R}_{i(6)}^{* 6}:=R^{\eta+1}$. Let $j(0), l(0) \in N \backslash\{1\}$ distinct such that $\left(R_{1}^{* 0}, R_{j(0)}^{* 0}, R_{l(0)}^{* 0}\right) \in \mathcal{L}$. Let $i(1) \notin$ $\{1, j(0), l(0)\}$. Let $i(2):=1$. Let $j(2), l(2) \in N$ be such that $\left(R^{0}, R_{j(2)}^{* 2}, R_{l(2)}^{* 2}\right) \in$ $\mathcal{L}$. Let $i(3) \notin\{1, j(2), l(2)\}$. Let $j(3), l(3)$ be two distinct elements of the set $\{j(2), l(2), i(3)\} \backslash\{2\}$. Let $i(4):=2$. Let $l(4) \in\{j(3), l(3)\} \backslash\{2\}$. Let $i(5) \notin\{1,2, l(4)\}$. Let $l(5) \in\{l(4), i(5)\} \backslash\{3\}$. Let $i(6):=3$. Let $j(1):=j(0), j(4):=2, j(4):=2, l(1):=l(0)$. Finally, let $g(k):=1$ for all $k \neq 1$ and let $g(1):=i(1)$. By construction, for each $k, R_{\{g(k), j(k), l(k)\}}^{* k} \in \mathcal{L}$. Comparing profile $R_{N}^{* k}$ and $R_{N}^{* k+1}$, the peak of agents $g(k), j(k)$ and $l(k)$ are kept fixed. By replacement-domination, agents $g(k), j(k)$ and $l(k)$ are all three affected in the same direction. Since $R_{\{g(k), j(k), l(k)\}}^{* k} \in \mathcal{L}$, this implies that $\Phi\left(R_{N}^{* k}\right)=\Phi\left(R_{N}^{* k+1}\right)$. Therefore, the sequence $\left\{\Phi\left(R_{N}^{* k}\right)\right\}_{0}^{6}$ is constant. In particular $\Phi\left(R_{N}^{* 0}\right)=\Phi\left(R_{N}^{* 6}\right)$. Since $\left(R_{1}^{* 6}, R_{2}^{* 6}, R_{3}^{* 6}\right) \in \mathcal{L}$, we also have by Step $0, \Phi\left(R_{N}^{* 6}\right)=\Phi\left(R^{0}, R^{\eta}, R^{\eta+1}, \ldots, R^{\eta+1}\right)=x_{\Phi}^{*}$. Since $R_{N}^{* 0}=R_{N}$, we obtain $\Phi\left(R_{N}\right)=x_{\Phi}^{*}$.

Case 2. $v \geq 4$ is even. Throughout the rest of the proof, let $x_{r}:=x_{r * r+v / 2}$, for all $r$. For all list $r_{1}, \ldots, r_{m}$, let $I\left(r_{1}, \ldots, r_{m}\right):=\left\{x \in \mathcal{C}: x I^{r_{1}} x_{r_{1}}, \ldots, x I^{r_{m}} x_{r_{m}}\right\}$. The proof is in a number of steps. Step a: Let $R_{N}$ be any profile, such that there exists $r$ such that that $R^{r}, R^{r+\frac{v}{2}} \in S\left(R_{N}\right)$. Clearly for all $M \subseteq N$, we have $E\left(R_{M}\right) \subseteq \mathcal{C}=E\left(R^{r}, R^{r+\frac{v}{2}}\right)$. Therefore by Step 1, we have $\Phi\left(R_{N}\right)$ $I^{r * r+v / 2} x_{r}$, that is $\Phi\left(R_{N}\right) I^{r} x_{r}$ holds for any such profile $R_{N}$. Step b: Let $R_{N}$ be any profile such that there are $i, j, k \in N$ satisfying $R_{\{i, j, k\}} \in \mathcal{L}$. Let $r$ be such that $R_{i}=R^{r}$. Let $u \neq i, j, k$. Let $R_{N}^{\prime}:=\left(R_{N \backslash\{u\}}, R_{u}^{r+v / 2}\right)$. By replacement-domination, we have $\Phi\left(R_{N}\right) I^{r} \Phi\left(R_{N}^{\prime}\right)$. Since $R_{N}^{\prime}$ satisfies the assumptions of Step a, we have $\Phi\left(R_{N}^{\prime}\right) I^{r} x_{r}$. Therefore, $\Phi\left(R_{N}\right) I^{r} x_{r}$ holds for 
any such profile $R_{N}$. Step c: For all $r$, the set $I(r, r+1)$ contains at most one element. Step d: Let $v \neq 4$. By step b, we have $\Phi\left(R^{r-1}, R^{r+1}, R^{r+\frac{v}{2}}, \ldots, R^{r+\frac{v}{2}}\right) \in$ $I\left(r-1, r+1, r+\frac{v}{2}\right)$. Since $I^{r}=I^{r+\frac{v}{2}}$ and, by Step a, $x_{r+\frac{v}{2}} I^{r} x_{r}$ it then follows that $\Phi\left(R^{r-1}, R^{r+1}, R^{r+\frac{v}{2}}, \ldots, R^{r+\frac{v}{2}}\right) \in I\left(r-1, r+1, r+\frac{v}{2}\right)$. This proves that for all $r$, the set $I(r-1, r, r+1)$ is nonempty. By step c, it contains at most one element, therefore it contains exactly one element. Thus for all $r$, both sets $I(r-1, r)$ and $I(r, r+1)$, which contain $I(r-1, r, r+1)$, are nonempty. By step c, both sets contain a unique element and are equal. Step e: If $v=4$, the set $I(0,1)$ contains at least $\Phi\left(R^{0}, R^{1}, R^{2}, R^{3}, \ldots, R^{3}\right)$, by Step a, and contains only this element, by step c. If $v \neq 4$, it immediately follows from Step d that the set $I(0, \ldots, v-1)$ is a singleton set. Therefore, in all cases, this set contains a unique element. Let $x_{\Phi}^{*}$ be this unique element. We now verify that $x_{\Phi}^{*}$ satisfies all the desired properties. Step f: Let $R_{N} \in \mathcal{P}$. Step a ensures that $\Phi\left(R_{N}\right) I_{N} x_{\Phi}^{*}$. Step g: Let $R_{N} \in \mathcal{I}$ such that $E\left(R_{N}\right)=\left[c_{r}, c_{r+\frac{v}{2}}\right]$. Again Step a ensures that $\Phi\left(R_{N}\right) I^{r} x_{\Phi}^{*}$. This and $\Phi\left(R_{N}\right) \in\left[c_{r}, c_{r+\frac{v}{2}}\right]$ (i.e. Pareto-efficiency) imply that $\Phi\left(R_{N}\right)=x_{\Phi}^{*}$ if $x_{\Phi}^{*} \in E\left(R_{N}\right)$ and $\Phi\left(R_{N}\right)=\sigma\left(x_{\Phi}^{*}\right)$ otherwise. Step h: Finally, let $R_{N} \in \mathcal{L}$. Either there are $r, s$ such that $s \notin\left\{r, r+\frac{v}{2}\right\}$ and $S\left(R_{N}\right)=\left\{R^{r}, R^{s}, R^{r+\frac{v}{2}}, R^{s+\frac{v}{2}}\right\}$, or there are $r, s, t$ such that $\left\{R^{r}, R^{s}, R^{t}\right\} \subseteq S\left(R_{N}\right)$ and $\left(R^{r}, R^{s}, R^{t}\right) \in \mathcal{L}$. In the first case, Step a implies that $\Phi\left(R_{N}\right) \in I(r, s)$. Since $s \notin\left\{r, r+\frac{v}{2}\right\}$, this implies $\Phi\left(R_{N}\right)=x_{\Phi}^{*}$. In the second case, by Step b, $\Phi\left(R_{N}\right) \in I(r, s, t)$. Again, since $\left(R^{r}, R^{s}, R^{t}\right) \in \mathcal{L}$, this implies that $\Phi\left(R_{N}\right)=x_{\Phi}^{*}$.

The following definitions plays a role in the next result and later in the paper. We say that two solutions with the same domain are Pareto-equivalent if, for all profile $R_{M}$ in the domain, the locations selected by each of these two solutions for profile $R_{M}$ are Pareto-indifferent for $R_{M}$. Also, for all positive integer $q>1$, and all $s_{1}, \ldots, s_{q} \in \mathbb{Z}$, let $R^{s_{1}, \ldots, s_{q}}$ and $I^{s_{1}, \ldots, s_{q}}$ respectively denote the weak Pareto-domination relation and the Pareto-indifference relation for the 
preferences $R^{s_{1}}, \ldots, R^{s_{q}}$. For example, $x R^{1,2} y \Leftrightarrow\left(x R^{1} y\right.$ and $\left.x R^{2} y\right)$.

Theorem 2 Let $\Phi$ be a solution on $\mathcal{R}_{v}^{N}$ that satisfies Pareto-efficiency and replacement-domination. There exists a solution $\Pi$ on $\mathcal{U}_{v}$ that satisfies populationmonotonicity ${ }^{6}$ and coincides with $\Phi$ on $\mathcal{R}_{v}^{N}$.

Proof. Define a solution $\Gamma$ as follows. Let $x_{\Phi}^{*}$ as defined in Lemma 4 . For all $R_{M} \in \mathcal{L} \cup \mathcal{P}$, let $\Gamma\left(R_{M}\right):=x_{\Phi}^{*}$. For all $R_{M}$ such that $E\left(R_{M}\right)=\left[c_{r}, c_{s}\right]$, let $\Gamma\left(R_{M}\right):=\Phi\left(R^{r}, R^{r+1}, R^{s}, \ldots, R^{s}\right)$. Let us verify that $\Gamma$ satisfies populationmonotonicity. We distinguish three cases. Let $R_{M} \in \mathcal{R}_{v}^{N}$ and $i \notin M, R_{i}^{\prime} \in \mathcal{R}_{v}$. Case 1. $E\left(R_{i}^{\prime}, R_{M}\right)=E\left(R_{M}\right)$. Then $\Gamma\left(R_{i}^{\prime}, R_{M}\right)=\Gamma\left(R_{M}\right)$ and populationmonotonicity is satisfied. Case 2. $v$ is even and $R_{i} \notin S\left(R_{M}\right)=\left\{R^{r}, R^{r+\frac{v}{2}}\right\}$. Then $E\left(R_{i}^{\prime}, R_{M}\right)$ equals either $\left[c_{r}, c_{r+\frac{v}{2}}\right]$ or $\left[c_{r+\frac{v}{2}}, c_{r}\right]$. Therefore, $\Gamma\left(R_{i}^{\prime}, R_{M}\right)$ is either equal to $\Phi\left(R^{r}, R^{r+1}, R^{r+\frac{v}{2}}, \ldots, R^{r+\frac{v}{2}}\right)$ or to $\Phi\left(R^{r+\frac{v}{2}}, R^{r+\frac{v}{2}+1}, R^{r}, \ldots, R^{r}\right)$. By Lemma 4 , in both cases, $\Gamma\left(R_{i}^{\prime}, R_{M}\right) I^{r, r+\frac{v}{2}} x_{\Phi}^{*}$. Since $\Gamma\left(R_{M}\right)=x_{\Phi}^{*}$, we obtain $\Gamma\left(R_{i}^{\prime}, R_{M}\right) I^{r, r+\frac{v}{2}} \Gamma\left(R_{M}\right)$, i.e. $\Gamma\left(R_{i}^{\prime}, R_{M}\right) I_{M} \Gamma\left(R_{M}\right)$ and populationmonotonicity is satisfied. Case 3. $E\left(R_{M}\right)=\left[c_{r}, c_{s}\right]$. Let $x:=\Phi\left(R_{N}^{r * s}\right), R_{n}^{\prime \prime}:=$ $R_{i}^{\prime}$ and $y:=\Phi\left(R_{N \backslash\{n\}}^{r * s}, R_{n}^{\prime \prime}\right)$. By replacement-domination, we have $x R^{r} y$ and $x R^{s} y$. These relations imply that for all $t$ such that $c_{t} \in\left[c_{r}, c_{s}\right]$, we have $x$ $R^{t} y$. Since $\Gamma\left(R_{M}\right)=x$ and $\Gamma\left(R_{M}, R_{i}^{\prime}\right)=y$, we obtain that for all such $t$, we have $\Gamma\left(R_{M}\right) R^{t} \Gamma\left(R_{M}, R_{i}^{\prime}\right)$. Since $S\left(R_{M}\right) \subseteq\left\{R^{t}: c_{t} \in\left[c_{r}, c_{s}\right]\right\}$, this implies $\Gamma\left(R_{M}\right) R_{M} \Gamma\left(R_{M}, R_{i}^{\prime}\right)$. Thus population-monotonicity is satisfied. Therefore, $\Gamma$ satisfies population-monotonicity on $\mathcal{U}_{v}$. By construction, the restriction of $\Gamma$ to $\mathcal{R}_{v}^{N}$ is Pareto-indifferent to $\Phi$. Let $\Pi$ be the solution such that $\Pi\left(R_{N}\right):=\Phi\left(R_{N}\right)$ on $\mathcal{R}_{v}^{N}$ and $\Pi\left(R_{M}\right):=\Gamma\left(R_{M}\right)$ for all $R_{M} \in \mathcal{U}_{v}$ such that $M \neq N$. Clearly $\Pi$ satisfies the desired properties.

\footnotetext{
${ }^{6}$ The construction of $\Pi$ further ensures that for all populations $M$, the restriction of $\Pi$ to $\mathcal{R}_{v}^{M}$ also satisfies replacement-domination.
} 


\subsection{Characterization results}

For values $v$ in $\{3,4,5\}$, solutions satisfying Pareto-efficiency and solidarity exist. We will show that they are all "priority rules", which we introduce next.

Throughout Sections 4.4 and 4.5, given a solution $\Pi$ that satisfies Paretoefficiency and population-monotonicity, we will use the following notation. For all population $M$, let $R_{M}^{* v}$ be the profile such that for all $i \in M$, agent $i$ has the preference $R_{i}^{* v}=R^{i} \in \mathcal{R}_{v}{ }^{7}$

\subsubsection{Efficient priority rules}

An efficient priority rule selects locations according to a priority ordering over locations, under the constraint of Pareto-efficiency.

A priority ordering $\succ$ is a binary relation over $\mathcal{C}$, that is transitive, irreflexive, asymmetric, and not necessarily complete, i.e. a strict partial order. Let $\succeq$ be the corresponding weak partial order such that for all $x, y \in \mathcal{C}$, we have $(x \succeq y) \Longleftrightarrow(x \succ y)$ or $(x=y)$.

A solution $\Pi$ on $\mathcal{U}_{v}$ is the efficient priority rule associated with the priority ordering $\succ$ if, for all $R_{M} \in \mathcal{U}_{v}$, we have $\Pi\left(R_{M}\right) \in E(R)$ and for all $R_{M} \in \mathcal{U}_{v}$ and all $x \in E\left(R_{M}\right)$, such that $x \neq \Pi\left(R_{M}\right)$, we have $\Pi\left(R_{M}\right) \succ x$. Let $\Pi_{\succ}$ be the priority rule associated with $\succ$. Similarly, a solution $\Phi$ on $\mathcal{R}_{v}^{N}$ is the efficient priority rule associated with the priority ordering $\succ$ if, for all $R_{N} \in \mathcal{R}_{v}^{N}$, we have $\Phi(R) \in E\left(R_{N}\right)$, and for all $R_{N} \in \mathcal{R}_{v}^{N}$ and all $x \in E\left(R_{N}\right)$, such that $x \neq \Phi\left(R_{N}\right)$, we have $\Phi\left(R_{N}\right) \succ x$. Let $\Phi_{\succ}$ be the priority rule associated with $\succ$. The following conditions (1) and (2) on $\succ$ and $v$ ensure that the maximum of $\succ$ is always unique on the Pareto-set of any preference profile in $\mathcal{U}$. They are therefore sufficient for either $\Pi_{\succ}$ or $\Phi_{\succ}$ to be well defined.

Condition (1) For all $y \in \mathcal{C}$, the set $\{z \in \mathcal{C}: z \succ y\}$ is finite.

\footnotetext{
${ }^{7}$ For example, $R_{\{1,4\}}^{* 5}$ is such that $R_{1}^{* 5}=R^{1} \in \mathcal{R}_{5}$ and $R_{4}^{* 5}=R^{4} \in \mathcal{R}_{5}$.
} 
Condition (2) For all $R_{M} \in \mathcal{U}_{v}$ (respectively $\mathcal{R}_{v}^{N}$ ), and all $y \neq z \in E\left(R_{M}\right)$, either $y \succ z$, or $z \succ y$, or there exists $x \in E\left(R_{M}\right)$ such that $x \succ y$ and $x \succ z$.

Well-defined efficient priority rules are obviously Pareto-efficient. They do not necessarily satisfy solidarity conditions. However, the rest of the analysis demonstrates that Pareto-efficient solutions that satisfy a solidarity condition are necessarily efficient priority rules. Moreover, solidarity imposes constraints on the relative position of the locations with highest priorities.

\subsubsection{The case $v=3$}

When $v=3$, all solutions that satisfy Pareto-efficiency and either populationmonotonicity or replacement-domination are efficient priority rules. Moreover, the same three-dimensional family of priorities characterize the solutions to both problems. A solution in this family is identified with a parameter $\delta=$ $(x, y, z) \in \mathcal{C}^{3}$, that satisfies, for some $s \in\{0,1,2\}$, the relations $x \in\left[c_{s}, c_{s+1}[\right.$, $y \in\left[c_{s+1}, c_{s+2}\right], z \in\left[c_{s+2}, c_{s}\right], y R^{s+1} x$ and $z R^{s} x$. Let $\Delta \subseteq \mathcal{C}^{3}$ be the set of such triples. For all $\delta \in \Delta$, let $\succ_{\delta}$ such that, for all $u \in \mathcal{C} \backslash\{x, y, z\}$, we have $x \succeq_{\delta} y \succ_{\delta} u$ and $x \succeq_{x} z \succ_{\delta} u$. Let $\Pi_{\delta}(R)$ be the priority rule on $\mathcal{U}_{3}$ associated with the priority $\succ_{\delta}$, and let $\Phi_{\delta}(R)$ be the efficient priority rule on $\mathcal{R}_{3}^{N}$ associated with $\succ_{\delta}$.

For example (see Fig. 1), let $\delta$ be a vector in $\Delta$ such that $s=2$. The rule $\Pi_{\delta}$ selects a location as follows. Let $R_{M}$ be any profile in $\mathcal{U}_{3}$. If $x$ is an element of $E\left(R_{M}\right)$, then the rule selects the location $x$. In the opposite case, at most one location among $y$ and $z$ belongs to $E\left(R_{M}\right)$. If one among these two locations does and $x$ does not, then the rule selects this location. Finally, if none of these three locations belong to $E\left(R_{M}\right)$, then $R_{M}$ must be a unanimous profile. If this is the case, the rule $\Pi_{x}$ selects the location commonly preferred by all agents in profile $R_{M}$. 


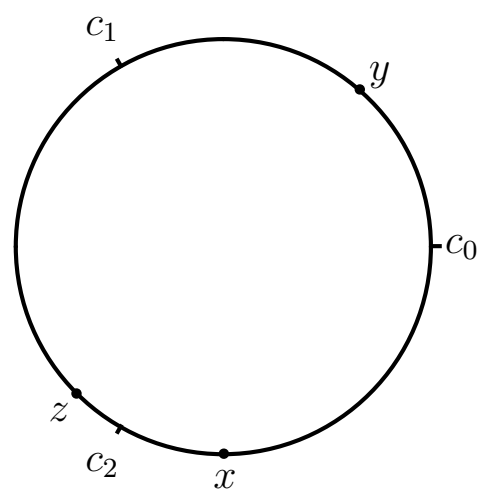

Figure 1: The case $v=3$. A triple $\delta=(x, y, z)$ in $\Delta$, such that $s=3$.

Theorem 3 (i) A solution on $\mathcal{U}_{3}$ satisfies Pareto-efficiency and populationmonotonicity iff it is in $\left\{\Pi_{\delta}\right\}_{\delta \in \Delta}$. (ii) A solution on $\mathcal{R}_{3}^{N}$ satisfies Paretoefficiency and replacement-domination iff it is in $\left\{\Phi_{\delta}\right\}_{\delta \in \Delta}$.

Proof of (i). $\Pi_{\delta}$ is well defined, since conditions (1) and (2) are clearly satisfied, for $\delta \in \Delta$.

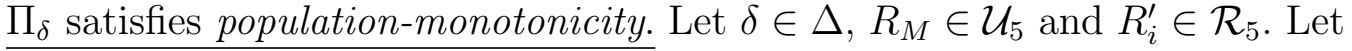
$\chi:=\Pi_{\delta}\left(R_{M}\right)$ and $\omega:=\Pi_{\delta}\left(R_{i}^{\prime}, R_{M}\right)$. We will show that population-monotonicity is satisfied, i.e. $\chi R_{M} \omega$. First, if $R_{M}$ is a unanimous profile, the axiom is trivially satisfied. Suppose through the end of the proof that $R_{M}$ is not unanimous. As a consequence, $\left(R_{i}^{\prime}, R_{M}\right)$ is not unanimous either. Therefore both $\chi$ and $\omega$ are elements of $\{x, y, z\}$. Since $E\left(R_{M}\right) \subseteq E\left(R_{i}^{\prime}, R_{M}\right)$, we have $\omega \succeq_{\delta} \chi$. Let us examine each case. I) Suppose that $\omega=\chi$. The axiom is trivially satisfied in this case. II) Suppose that $\omega=x, \chi=y$ and $x \neq y$. Then $S\left(R_{M}\right)=\left\{R^{s+1}, R^{s+2}\right\}$. Since $y R^{s+1, s+2} x$ holds, the axiom is satisfied. II) Suppose that $\omega=x, \chi=z$ and $x \neq z$. Then $S\left(R_{M}\right)=\left\{R^{s}, R^{s+2}\right\}$. Since $z R^{s, s+2} x$ holds, the axiom is satisfied. Since $\omega \succeq_{\delta} \chi, x \succeq_{\delta} y, x \succeq_{\delta} z$ and $\chi, \omega \in\{x, y, z\}$, there are no other 
cases, which ends the proof.

If $\Pi$ satisfies Pareto-efficiency and population-monotonicity, then $\Pi$ equals $\Pi_{\delta}$ for some $\delta \in \Delta$. Let $\Pi$ be a solution on $\mathcal{U}_{3}$ satisfying Pareto-efficiency and population-monotonicity. For all population $M$, let $\theta_{M}:=\Pi\left(R_{M}^{* 3}\right)$. Let $x:=$ $\theta_{\{0,1,2\}}$. Let $s$ be the unique index in $\{0,1,2\}$ such that $x \in\left[c_{s}, c_{s+1}[\right.$. Let $y:=$ $\theta_{\{s+1, s+2\}}$ and $z:=\theta_{\{s+2, s\}}$. By population-monotonicity ${ }_{+}$, applied to profiles $R_{\{0,1,2\}}$ and $R_{\{s+1, s+2\}}$, we have $y R^{s+1} x$. By population-monotonicity ${ }_{+}$, applied to profiles $R_{\{0,1,2\}}$ and $R_{\{s+2, s\}}$, we have $z R^{s+1} x$. Thus $\delta:=(x, y, z) \in \Delta$. It remains to show that $\Pi=\Pi_{\delta}$. Since $v=3$ is odd, there are no profiles in $\mathcal{P}$. Lemma 3 implies that $\Pi\left(R_{M}\right)$ only depends on $E\left(R_{M}\right)$. Let $R_{M} \in \mathcal{U}_{3}$ such that $x \in E\left(R_{M}\right)$. By Lemma 3 applied to $R_{\{0,1,2\}}$ and $R_{M}$, we have $\Pi\left(R_{M}\right)=\theta_{\{0,1,2\}}=x=\Pi_{\delta}\left(R_{M}\right)$. Let $R_{M} \in \mathcal{U}_{3}$ be a non unanimous profile such that $x \notin E\left(R_{M}\right)$. Then either $E\left(R_{M}\right)=\left[c_{s+1}, c_{s+2}\right]$ or $E\left(R_{M}\right)=\left[c_{s+2}, c_{s}\right]$. Suppose that $E\left(R_{M}\right)=\left[c_{s+1}, c_{s+2}\right]$. Since $\Pi$ only depends on the Pareto-set and $E\left(R_{M}\right)=E\left(R_{\{s+1, s+2\}}\right)$, we have $\Pi\left(R_{M}\right)=\theta_{\{s+1, s+2\}}=y=\Pi_{\delta}\left(R_{M}\right)$. Suppose instead $E\left(R_{M}\right)=\left[c_{s+2}, c_{s}\right]$. Since $\Pi$ only depends on the Pareto-set and $E\left(R_{M}\right)=E\left(R_{\{s, s+2\}}\right)$, we have $\Pi\left(R_{M}\right)=\theta_{\{s, s+2\}}=z=\Pi_{\delta}\left(R_{M}\right)$. Since $\Pi$ and $\Pi_{\delta}$ coincide on any non-unanimous profile, we proved that $\Pi=\Pi_{\delta}$.

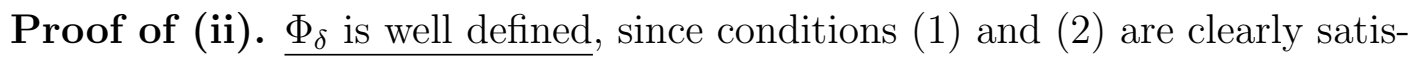
fied, for $\delta \in \Delta$.

$\Phi_{\delta}$ satisfies replacement-domination. Let $\delta \in \Delta, i \in N, L:=N \backslash\{i\}, R_{L} \in$ $\mathcal{R}_{3}^{L}$ and $R_{i}, R_{i}^{\prime} \in \mathcal{R}_{3}$. Let $\omega:=\Phi_{\delta}\left(R_{i}, R_{L}\right)$ and $\omega^{\prime}:=\Phi_{\delta}\left(R_{i}^{\prime}, R_{L}\right)$. We will show that replacement-domination is satisfied, i.e. either $\omega R_{L} \omega^{\prime}$, or $\omega^{\prime} R_{L} \omega$. Observe that we also have $\omega=\Pi_{\delta}\left(R_{i}, R_{L}\right)$ and $\omega^{\prime}=\Pi_{\delta}\left(R_{i}^{\prime}, R_{L}\right)$ and that $\Pi_{\delta}$ satisfies population-monotonicity. Let $\chi:=\Pi_{\delta}\left(R_{L}\right)$. First, if $R_{L}$ is a unanimous profile, the axiom is trivially satisfied. Suppose through the end of the proof that this is not the case, which also implies that $\left(R_{i}, R_{L}\right)$ and $\left(R_{i}^{\prime}, R_{L}\right)$ are not unanimous either. Therefore, $\omega, \omega^{\prime}, \chi \in\{x, y, z\}$. Second, if $\omega=\omega^{\prime}$, the axiom 
is trivially satisfied. Suppose through the end of the proof that $\omega \neq \omega^{\prime}$. This implies in particular that at least one location among $\omega$ and $\omega^{\prime}$ differs from $x$. Suppose for example that $\omega \neq x$. We then have $\omega \in\{y, z\}$, which in turn implies $\chi=\omega$. By population-monotonicity, we then have $\chi R_{L} \omega^{\prime}$, therefore $\omega$ $R_{L} \omega^{\prime}$. If instead we suppose $\omega^{\prime} \neq x$, we obtain that $\omega^{\prime} R_{L} \omega$. In both cases, the axiom is satisfied, which ends the proof.

If $\Phi$ satisfies Pareto-efficiency and replacement-domination, then $\Phi$ equals $\Phi_{\delta}$ for some $\delta \in \Delta$. Let $\Phi$ be a solution on $\mathcal{R}_{3}^{N}$ satisfying these two conditions. By Theorem 2, there exist a solution $\Pi$ on $\mathcal{U}_{3}$ that coincides with $\Phi$ on $\mathcal{R}_{3}^{N}$ and satisfies Pareto-efficiency and population-monotonicity. By (i), there exists $\delta \in \Delta$ such that $\Pi=\Pi_{\delta}$, which implies that $\Phi=\Phi_{\delta}$, with $\delta \in \Delta$.

\subsubsection{The case $v=4$}

When $v=4$, the solutions satisfying the conditions of Pareto-efficiency and population-monotonicity form a unidimensional family of efficient priority rules. Only four of these solutions satisfy Pareto-efficiency and replacement-domination. They are also priority rules, associated with priorities selected from the first family.

Let us introduce these families. A priority in the first family is identified with a parameter $x \in \mathcal{C}$. For any such $x$, let $s$ be the unique index in $\{0, \ldots, 3\}$ such that $x \in\left[c_{s}, c_{s+1}\left[\right.\right.$. Let $\succ_{x}$ such that $x \succeq_{x} \sigma_{s}(x) \succ_{x} \sigma(x) \succ_{x} y$ and $x \succ_{x} \sigma_{s+1}(x) \succeq_{x} \sigma(x) \succ_{x} y$, for all $y \in \mathcal{C} \backslash\left\{x, \sigma_{s}(x), \sigma_{s+1}(x), \sigma(x)\right\}$. Let $\Pi_{x}\left(R_{M}\right)$ be the efficient priority rule on $\mathcal{U}_{4}$ associated with the priority $\succ_{x}$.

For example (see Fig. 2), let $x$ be a location in $\left[c_{2}, c_{3}[\right.$. Then $s=2$. The rule $\Pi_{x}$ selects a location as follows. Let $R_{M}$ be any profile in $\mathcal{U}_{4}$. If $x$ is an element of $E\left(R_{M}\right)$, then the rule selects the location $x$. In the opposite case, at most one location among $\sigma_{2}(x)$ and $\sigma_{3}(x)$ belongs to $E\left(R_{M}\right)$. If one among these two locations does, then the rule selects this location. If neither $x, \sigma_{2}(x)$ nor 


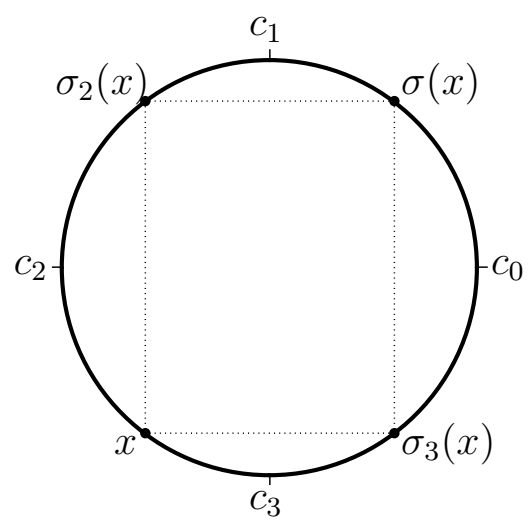

Figure 2: The case $v=4$. A location $x$ such that $s=2$ and its symmetric images $\sigma(x), \sigma_{2}(x)$ and $\sigma_{3}(x)$.

$\sigma_{3}(x)$ belong to $E\left(R_{M}\right)$, but $\sigma(x)$ does, then the rule selects the location $\sigma(x)$. Finally, if none of these four locations belong to $E\left(R_{M}\right)$, then $R_{M}$ must be a unanimous profile. If this is the case, the rule $\Pi_{x}$ selects the location commonly preferred by all agents in profile $R_{M}$.

A priority in the second family is identified with an index $s \in\{0, \ldots, 3\}$. Let $\succ_{s}$ such that for all $y \in \mathcal{C} \backslash\left\{c_{s}, c_{s+2}\right\}$, we have $c_{s} \succ_{s} c_{s+2} \succ_{s} y$. Let $\Phi_{s}$ be the efficient priority rule on $\mathcal{R}_{4}^{N}$ associated with the priority $\succ_{s}$. Observe that $\succ_{s}=\succ_{c_{s}}$ so that $\Phi_{s}$ is in fact the restriction of $\Pi_{c_{s}}$ to $\mathcal{R}_{4}^{N}$.

Theorem 4 (i) A solution on $\mathcal{U}_{4}$ satisfies Pareto-efficiency and populationmonotonicity iff it is Pareto-equivalent to a solution in $\left\{\Pi_{x}\right\}_{x \in \mathcal{C}}$. (ii) A solution on $\mathcal{R}_{4}^{N}$ satisfies Pareto-efficiency and replacement-domination iff it is Paretoequivalent to a solution in $\left\{\Phi_{0}, \ldots, \Phi_{3}\right\}$.

Proof of (i). $\quad \Pi_{x}$ is well defined. Condition (1) is clearly satisfied, for all $x \in \mathcal{C}$. Condition $(2)$ is obviously satisfied for all pairs $(y, z)$ that are comparable for the priority $\succ_{x}$. The only pair that is not necessarily comparable is 
$\left(\sigma_{s}(x), \sigma_{s+1}(x)\right)$. But for all values of $x$ such that this is the case, the location $x$ itself is in the Pareto-set for any profile $R_{M}$ such that $E\left(R_{M}\right)$ contains them both. Moreover $x$ has a higher priority rank than both of these locations for the priority $\succ_{x}$. Therefore condition (2) holds and $\Pi_{x}$ is well defined, for all $x \in \mathcal{C}$.

$\Pi_{x}$ satisfies population-monotonicity. We leave it to the reader to verify that for all $x \in \mathcal{C}$, we have $\sigma_{s+1}(x) R^{s+2} x, \sigma_{s+1}(x) I^{s+1, s+3} x, \sigma_{s}(x) R^{s+3} x, \sigma_{s}(x)$ $I^{s, s+2} x, \sigma(x) R^{s+2, s+3} \sigma_{s+1}(x)$ and $\sigma(x) R^{s+2, s+3} \sigma_{s}(x)$. Let $x \in \mathcal{C}, R_{M} \in \mathcal{U}_{4}$ and $R_{i}^{\prime} \in \mathcal{R}_{4}$. Let $\chi:=\Pi_{\lambda}\left(R_{M}\right)$ and $\omega:=\Pi_{\lambda}\left(R_{i}^{\prime}, R_{M}\right)$. We will show that population-monotonicity is satisfied, i.e. $\chi R_{M} \omega$. As a starting point, observe that if either $R_{M}$ is a unanimous profile, or if $\chi=\omega$, the axiom is trivially satisfied. Thus we can restrict attention to the remaining cases. Suppose through the end of the proof that $R_{M}$ is not unanimous, and that $\chi \neq \omega$. As a consequence, $\left(R_{i}^{\prime}, R_{M}\right)$ is not unanimous either. All remaining cases are such that either $\left(\omega=x\right.$ and $\left.\chi \in\left\{\sigma_{s}(x), \sigma_{s+1}(x)\right\}\right)$ or $\left(\omega \in\left\{\sigma_{s}(x), \sigma_{s+1}(x)\right\}\right.$ and $\chi \in\{\sigma(x), x\})$. Let us examine each case. I) Suppose that ( $\omega=x$ and $\left.\chi=\sigma_{s+1}(x)\right)$. Then $S\left(R_{M}\right) \subseteq\left\{R^{s+1}, R^{s+2}, R^{s+3}\right\}$. Since $\sigma_{s+1}(x) R^{s+1, s+2, s+3}$ $x$ holds, the axiom is satisfied. II) Suppose that $\left(\omega=x\right.$ and $\left.\chi=\sigma_{s}(x)\right)$. Then $S\left(R_{M}\right) \subseteq\left\{R^{s+2}, R^{s+3}, R^{s}\right\}$. Since $\sigma_{s}(x) R^{s, s+2, s+3} x$ holds, the axiom is satisfied. III) Suppose that $\left(\omega \in\left\{\sigma_{s+1}(x), \sigma_{s}(x)\right\}\right.$ and $\left.\chi=\sigma(x)\right)$. Then $S\left(R_{M}\right)=\left\{R^{s+2}, R^{s+3}\right\}$. Since $\sigma(x) R^{s+2, s+3} \sigma_{s+1}(x)$ and $\sigma(x) R^{s+2, s+3} \sigma_{s}(x)$ hold, the axiom is satisfied. $I V)$ Suppose that $\left(\omega=\sigma_{s+1}(x)\right.$ and $\left.\chi=x\right)$. Then $S\left(R_{M}\right)=\left\{R^{s+1}, R^{s+3}\right\}$. Since $x R^{s+1, s+3} \sigma_{s+1}(x)$ holds, the axiom is satisfied. $V)$ Suppose that $\left(\omega=\sigma_{s}(x)\right.$ and $\left.\chi=x\right)$. Then $S\left(R_{M}\right)=\left\{R^{s}, R^{s+2}\right\}$. Since $x R^{s, s+2} \sigma_{s}(x)$ holds, the axiom is satisfied. We exhausted all possible cases, which ends the proof.

If $\Pi$ satisfies Pareto-efficiency and population-monotonicity, then, for some

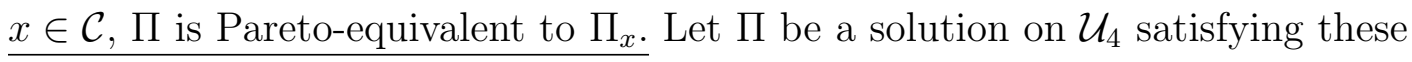
two conditions. For all population $M$, let $\theta_{M}:=\Pi\left(R_{M}^{* 4}\right)$. Let $x:=\theta_{\{0,1,2,3\}}$. 
Let $s$ be the unique index in $\{0, \ldots, 3\}$ such that $x \in\left[c_{s}, c_{s+1}[\right.$. We now verify that necessarily $\Pi$ is Pareto-equivalent to $\Pi_{x}$. Let $R_{M}$ be a non unanimous profile. We distinguish several cases. Case 1: Suppose first that $E\left(R_{M}\right)$ contains $x$. Then Lemma 3 ensures that $\Pi\left(R_{M}\right) I_{M} x=\Pi_{x}\left(R_{M}\right)$. Case 2: Suppose that $E\left(R_{M}\right)=\left[c_{s+1}, c_{s+3}\right]$. Consider profiles $R_{M}$ and $\left(R_{M}, R_{i}^{s}\right)$. By populationmonotonicity $_{+}$, we have $\Pi\left(R_{M}\right) R^{s+1} \Pi\left(R_{M}, R_{i}^{s}\right)$. Since $\Pi\left(R_{M}, R_{i}^{s}\right)=x$ and $\Pi\left(R_{M}\right) \in\left[c_{s+1}, c_{s+3}\right]$, we obtain $\Pi\left(R_{M}\right)=\sigma_{s+1}(x)=\Pi_{x}\left(R_{M}\right)$. Case 3: Suppose that $E\left(R_{M}\right)=\left[c_{s+2}, c_{s}\right]$. Consider profiles $R_{M}$ and $\left(R_{M}, R_{i}^{s+1}\right)$. By population-monotonicity ${ }_{+}$, we have $\Pi\left(R_{M}\right) R^{s} \Pi\left(R_{M}, R_{i}^{s+1}\right)$. Since we have $\Pi\left(R_{M}, R_{i}^{s+1}\right)=x$ and $\Pi\left(R_{M}\right) \in\left[c_{s+2}, c_{s}\right]$, we obtain $\Pi\left(R_{M}\right)=\sigma_{s}(x)=$ $\Pi_{x}\left(R_{M}\right)$. Case 4: Suppose that $E\left(R_{M}\right)=\left[c_{s+2}, c_{s+3}\right]$. Consider the profiles $R_{M}$ and $\left(R_{M}, R_{i}^{s+1}\right)$. By population-monotonicity $y_{+}$, we have $\Pi\left(R_{M}\right) R^{s+2}$ $\Pi\left(R_{M}, R_{i}^{s+1}\right)$. By Case 2 , we have $\Pi\left(R_{M}, R_{i}^{s+1}\right)=\sigma_{s+1}(x)$. Therefore, $\Pi\left(R_{M}\right)$ $R^{s+2} \sigma_{s+1}(x)$. Consider the profiles $R_{M}$ and $\left(R_{M}, R_{i}^{s}\right)$. Population-monotonicity requires that $\Pi\left(R_{M}\right) R^{s+3} \Pi\left(R_{M}, R_{i}^{s}\right)$. By Case 3, we have $\Pi\left(R_{M}, R_{i}^{s}\right)=$ $\sigma_{s}(x)$. Therefore, $\Pi\left(R_{M}\right) R^{s+3} \sigma_{s}(x)$. Finally, $\Pi\left(R_{M}\right) R^{s+2} \sigma_{s+1}(x)$ and $\Pi\left(R_{M}\right)$ $R^{s+3} \sigma_{s}(x)$ imply $\Pi\left(R_{M}\right)=\sigma(x)=\Pi_{x}\left(R_{M}\right)$. Last, $\Pi$ and $\Pi_{x}$ obviously coincide on all unanimous profiles, therefore we have shown that $\Pi$ is Paretoequivalent to $\Pi_{x}$ for some $x \in \mathcal{C}$.

Proof of (ii). $\Phi_{s}$ satisfies replacement-domination. Let $s \in\{0, \ldots, 3\}$. The rule $\Phi_{s}$ is obviously well defined and Pareto-efficient. Let $\lambda \in \mathcal{C}, i \in N, L:=N \backslash\{i\}$, $R_{L} \in \mathcal{R}_{4}^{L}$ and $R_{i}, R_{i}^{\prime} \in \mathcal{R}_{4}$. Let $\omega:=\Phi_{s}\left(R_{i}, R_{L}\right)$ and $\omega^{\prime}:=\Phi_{s}\left(R_{i}^{\prime}, R_{L}\right)$. Also, let $\chi:=\Pi_{c_{s}}\left(R_{L}\right)$. We will show that replacement-domination is satisfied, i.e. we either have $\omega R_{L} \omega^{\prime}$, or $\omega^{\prime} R_{L} \omega$. First, if $R_{L}$ is a unanimous profile, then the axiom is trivially satisfied. Thus we can restrict attention to the remaining cases. Suppose through the end of the proof that $R_{L}$ is not unanimous. This in turn implies that both $\left(R_{i}, R_{L}\right)$ and $\left(R_{i}^{\prime}, R_{L}\right)$ are not unanimous. Therefore $\left\{\omega, \omega^{\prime}, \chi\right\} \subseteq\left\{c_{s}, c_{s+2}\right\}$. Observe that we have $\omega=\Pi_{c_{s}}\left(R_{i}, R_{L}\right)$ and 
$\omega^{\prime}=\Pi_{c_{s}}\left(R_{i}^{\prime}, R_{L}\right)$. By population-monotonicity of $\Pi_{c_{s}}$, we have $\chi R_{L} \omega$ and $\chi$ $R_{L} \omega^{\prime}$. Two cases arise. First, if $\chi \in\left\{\omega, \omega^{\prime}\right\}$, then we have either $\omega R_{L} \omega^{\prime}$ or $\omega R_{L} \omega^{\prime}$. Therefore, in this case, the axiom is satisfied. Second, if $\chi \notin\left\{\omega, \omega^{\prime}\right\}$, since $\left\{\omega, \omega^{\prime}, \chi\right\} \subseteq\left\{c_{s}, c_{s+2}\right\}$, then necessarily $\omega=\omega^{\prime}$, and the axiom is trivially satisfied. This end the proof.

If $\Phi$ satisfies the conditions of Pareto-efficiency and replacement-domination then $\Phi$ is Pareto-equivalent to a solution in $\left\{\Phi_{0}, \ldots, \Phi_{3}\right\}$. Let $\Phi$ be a solution on $\mathcal{R}_{4}^{N}$ that satisfies these two conditions. By Theorem 2, there exist a solution $\Pi$ on $\mathcal{U}_{4}$ that coincides with $\Phi$ on $\mathcal{R}_{4}^{N}$ and satisfies Pareto-efficiency and population-monotonicity. By (i), there exists $x \in \mathcal{C}$ such that $\Pi$ is Paretoequivalent to $\Pi_{x}$. As before, let $s$ be the unique index in $\{0, \ldots, 3\}$ such that $x \in$ $\left[c_{s}, c_{s+1}\left[\right.\right.$. Let us show that $x=c_{s}$. Consider profiles $\left(R^{s+1}, R^{s+2}, R^{s+3}, \ldots, R^{s+3}\right)$ and $\left(R^{s}, R^{s+2}, R^{s+3}, \ldots, R^{s+3}\right)$, both in $\mathcal{R}_{4}^{N}$. By (i), we know that the equalities $\Phi\left(R^{s+1}, R^{s+2}, R^{s+3}, \ldots, R^{s+3}\right)=\sigma_{s+1}(x)$ and $\Phi\left(R^{s}, R^{s+2}, R^{s+3}, \ldots, R^{s+3}\right)=$ $\sigma_{s}(x)$ hold. Since $x \in\left[c_{s}, c_{s+1}\left[\right.\right.$, therefore $\left.\left.\sigma_{s+1}(x) \in\right] c_{s+1}, c_{s+2}\right]$ and $\sigma_{s}(x) \in$ ]$\left.c_{s+3}, c_{s}\right]$. Therefore, $\sigma_{s+1}(x) P^{s+2} \sigma_{s}(x)$, and replacement-domination requires that $\sigma_{s+1}(x) R^{s+3} \sigma_{s}(x)$, which in turn implies $x=c_{s}$. Therefore, $\Phi$ is Paretoequivalent to the restriction of $\Pi_{c_{s}}$ to $\mathcal{R}_{4}^{N}$, which is $\Phi_{s}$

\subsubsection{The case $v=5$}

When $v=5$, the solutions satisfying the conditions of Pareto-efficiency and population-monotonicity form a five-dimensional family of efficient priority rules. The solutions that satisfy Pareto-efficiency and replacement-domination form a one-dimensional family of efficient priority rules. As in previous cases, the family of priorities characterizing the second problem is a subset of the family of priorities characterizing the first problem.

Let us introduce these families. A solution in the first family is identified with a parameter $\lambda=(x, a, b, d, e)$ from a certain subset of $\mathcal{C}^{5}$, which we describe 
next. For any index $t \in\{0, \ldots, 4\}$, define the following sets of such vectors.

Let $\Lambda_{A}^{t}$ be the set of vectors $\lambda$ in $\mathcal{C}^{5}$ such that $\left.\left.x \in\left[c_{t+2}, c_{t-2}\right], a \in\right] c_{t+1}, c_{t+2}\right]$, $b \in\left[c_{t-2}, c_{t-1}\left[, d \in\left[c_{t-1}, c_{t+1}\right], b R^{t-2} x, a R^{t+2} x, d R^{t-1, t+1} x, e R^{t} d\right.\right.$, and in addition, the relations $d R^{t-1} b$ and $e R^{t+1} a$ hold. ${ }^{8}$

Let $\Lambda_{B}^{t}$ be the set of vectors $\lambda$ in $\mathcal{C}^{5}$ such that $\left.\left.x \in\left[c_{t+2}, c_{t-2}\right], a \in\right] c_{t+1}, c_{t+2}\right]$, $b \in\left[c_{t-2}, c_{t-1}\left[, d \in\left[c_{t-1}, c_{t+1}\right], b R^{t-2} x, a R^{t+2} x, d R^{t-1, t+1} x, e R^{t} d\right.\right.$, and in addition, the relations $d R^{t+1} a$ and $e R^{t-1} b$ hold. ${ }^{9}$

Let $\Lambda:=\cup_{t=0, \ldots, 4}\left(\Lambda_{A}^{t} \cup \Lambda_{B}^{t}\right)$ and $\Lambda_{\cap}:=\cup_{t=0, \ldots, 4}\left(\Lambda_{A}^{t} \cap \Lambda_{B}^{t}\right)$. Equivalently, a vector $\lambda$ is in $\Lambda_{\cap}$ if there is an index $t$ satisfying $x \in\left[c_{t+2}, c_{t-2}\right], a=c_{t+2}$, $b=c_{t-2}$ and $d=e=c_{t}$. Observe that for all $\lambda \in \Lambda$, the index $t$ is uniquely defined, in the sense that $t \neq t^{\prime} \Rightarrow\left(\Lambda_{A}^{t} \cup \Lambda_{B}^{t}\right) \cap\left(\Lambda_{A}^{t^{\prime}} \cup \Lambda_{B}^{t^{\prime}}\right)=\emptyset$.

For all $\lambda \in \Lambda$, let $\succ_{\lambda}$ such that $x \succeq_{\lambda} a \succ_{\lambda} d, x \succeq_{\lambda} b \succ_{\lambda} d$ and $d \succeq_{\lambda} e \succ_{\lambda} u$ for all $u$ such that $u \in \mathcal{C} \backslash\{x, a, b, d, e\}$. Let $\Pi_{\lambda}\left(R_{M}\right)$ be the efficient priority rule on $\mathcal{U}_{5}$, and let $\Phi_{\lambda}\left(R_{M}\right)$ be the efficient priority rule on $\mathcal{R}_{5}^{N}$ associated with the priority $\succ_{\lambda}$.

For example, let $\lambda=(x, a, b, d, e) \in \Lambda$ (see Fig. 3a). The rule $\Pi_{\lambda}$ selects a location as follows. Let $R_{M}$ be any profile in $\mathcal{U}_{5}$. If $x$ belongs to $E\left(R_{M}\right)$, then the rule selects $x$. In the opposite case, at most one location among $a$ and $b$ belongs to $E\left(R_{M}\right)$. If one among these two locations does and $x$ does not, then the rule selects this location. If neither $x, a$ nor $b$ belong to $E\left(R_{M}\right)$, but $d$ does, then the rule selects the location $d$. If neither $x, a, b$, nor $d$ belong to $E\left(R_{M}\right)$, but $e$ does, then the rule selects the location $e$. Finally, if none of these five locations belong to $E\left(R_{M}\right)$, then $R_{M}$ must be a unanimous profile. In this case, the rule $\Pi_{\lambda}$ selects the location commonly preferred by all agents in profile $R_{M}$.

\footnotetext{
${ }^{8}$ In particular, $d R^{t-1} b, b \in\left[c_{t-2}, c_{t-1}\left[\right.\right.$ and $d \in\left[c_{t-1}, c_{t+1}\right]$ imply $d \in\left[c_{t-1}, c_{t}\right]$. Similarly, e $R^{t} d$, e $R^{t+1} a, d \in\left[c_{t-1}, c_{t}\right]$ and $\left.\left.a \in\right] c_{t+1}, c_{t+2}\right]$ imply $e \in\left[c_{t}, c_{t+1}\right]$.

${ }^{9}$ In particular, $d R^{t+1} a, a \in\left[c_{t+1}, c_{t+2}\left[\right.\right.$ and $d \in\left[c_{t-1}, c_{t+1}\right]$ imply $d \in\left[c_{t}, c_{t+1}\right]$. Similarly, $e R^{t} d, e R^{t-1} b, d \in\left[c_{t}, c_{t+1}\right]$ and $\left.\left.b \in\right] c_{t-2}, c_{t-1}\right]$ imply $e \in\left[c_{t-1}, c_{t}\right]$.
} 
(a)

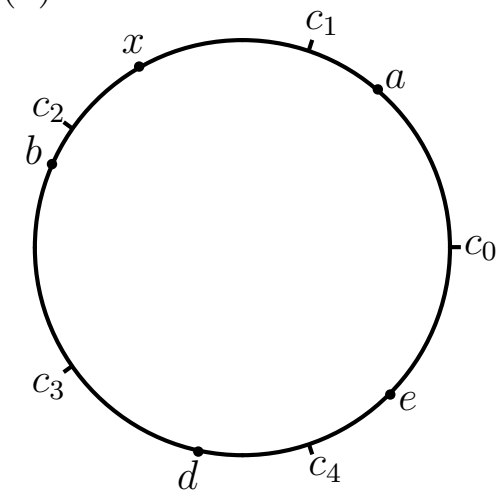

(b)

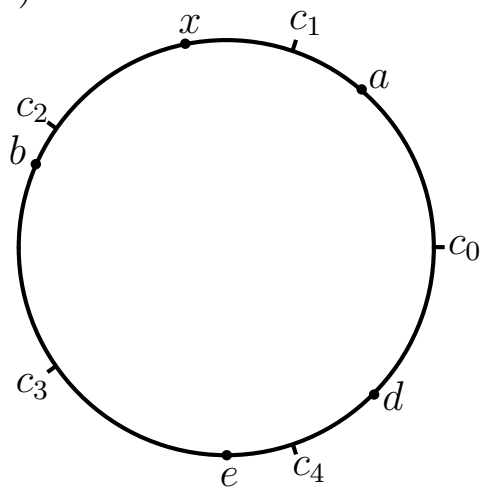

Figure 3: An illustration of the components of the parameter set $\Lambda$ when $v=5$. (a) A vector $\lambda=(x, a, b, d, e)$ in $\Lambda_{A}^{4}$. (b) A vector $\lambda=(x, a, b, d, e)$ in $\Lambda_{B}^{4}$.

Theorem $5(i)$ A solution on $\mathcal{U}_{5}$ satisfies Pareto-efficiency and population monotonicity iff it is in $\left\{\Pi_{\lambda}\right\}_{\lambda \in \Lambda}$. (ii) A solution on $\mathcal{R}_{5}^{N}$ satisfies Pareto-efficiency and replacement-domination iff it is in $\left\{\Phi_{\lambda}\right\}_{\lambda \in \Lambda_{\cap}}$.

Proof of $(\mathbf{i}) . \Pi_{\lambda}$ is well defined. Let $\lambda \in \Lambda$ and $R_{M} \in \mathcal{U}_{5}$. Let us prove that $\Pi_{\lambda}\left(R_{M}\right)$ is well defined. Condition (1) is clearly satisfied for all $\lambda$ in this set. Let us show that condition (2) is also always satisfied. This is trivially the case if $E\left(R_{M}\right)$ is a singleton. Suppose not. Then the definition of $\Lambda$ ensures that $E\left(R_{M}\right)$ contains at least one of the locations $x, a, b, d$ or $e$, which all have a higher priority than all remaining locations in $\mathcal{C}$. All pairs of locations among these five locations distinct from the pair $(a, b)$ are comparable. Thus, for these pairs, conditions (2) holds. We also have $a, b \in E\left(R_{M}\right) \Rightarrow x \in E\left(R_{M}\right), x \succeq_{\lambda} a$ and $x \succeq_{\lambda} b$. Therefore, condition (2) holds for the pair $(a, b)$ as well. Therefore, $\Pi_{\lambda}$ is well defined.

$\Pi_{\lambda}$ satisfies population-monotonicity. We let the reader verify that for all $\lambda \in \Lambda$, we have $a R^{t, t+1, t+2} x, b R^{t-2, t-1, t} x, d R^{t-1, t, t+1} x$ and $e R^{t-1, t, t+1} x$. 
In addition, if $\lambda \in \Lambda_{A}$, then $e R^{t, t+1} a, d R^{t-1, t} b$ and $e R^{t, t+1} d$. Similarly, if $\lambda \in \Lambda_{B}$, then $d R^{t, t+1} a, e R^{t-1, t} b$ and $e R^{t-1, t} d$. All these relations can be easily derived from the definitions. Let $\lambda \in \Lambda, R_{M} \in \mathcal{U}_{5}$ and $R_{i}^{\prime} \in \mathcal{R}_{5}$. Let $\chi:=$ $\Pi_{\lambda}\left(R_{M}\right)$ and $\omega:=\Pi_{\lambda}\left(R_{i}^{\prime}, R_{M}\right)$. We will show that population-monotonicity is satisfied, i.e. $\chi R_{M} \omega$. First, if $R_{M}$ is a unanimous profile, the axiom is trivially satisfied. Suppose through the end of the proof that $R_{M}$ is not unanimous. As a consequence, $\left(R_{i}^{\prime}, R_{M}\right)$ is not unanimous either. Therefore both $\chi$ and $\omega$ are elements of $\{x, a, b, d, e\}$. Second, if $\chi=\omega$, again the axiom is trivially satisfied. Suppose through the end of the proof that $\chi \neq \omega$. Since $E\left(R_{M}\right) \subset E\left(R_{i}^{\prime}, R_{M}\right)$, we have $\omega \succ_{\lambda} \chi$. Since $x, a, b, d \succ_{\lambda} e$, it follows that $\omega \in\{x, a, b, d\}$. Let us examine each case. I) Suppose that $\omega=x$, so that $\chi \in\{a, b, d, e\}$. If $\chi=a$, then $S\left(R_{M}\right) \subseteq\left\{R^{t}, R^{t+1}, R^{t+2}\right\}$. Similarly, if $\chi=b$, then $S\left(R_{M}\right) \subseteq\left\{R^{t-2}, R^{t-1}, R^{t}\right\}$. Finally, if $\chi \in\{d, e\}$, then $S\left(R_{M}\right) \subseteq\left\{R^{t-1}, R^{t}, R^{t+1}\right\}$. In the first case, we have $a R^{t, t+1, t+2} x$. In the second case, we have $b R^{t-2, t-1, t} x$. In the third case, we have $d R^{t-1, t, t+1} x$ and $e R^{t-1, t, t+1} x$. In all three cases, the axiom is therefore satisfied. II) Suppose that $\omega=a$. This implies that $S\left(R_{M}\right)=\left\{R^{t}, R^{t+1}\right\}$. Moreover, either $\left(\chi=e\right.$ and $\left.\lambda \in \Lambda_{A}\right)$, or $\left(\chi=d\right.$ and $\left.\lambda \in \Lambda_{B}\right)$. In both cases, we have $\chi R^{t, t+1} a$, i.e. the axiom is satisfied. III) Suppose that $\omega=b$. This implies that $S\left(R_{M}\right)=\left\{R^{t-1}, R^{t}\right\}$. Moreover, either $\left(\chi=d\right.$ and $\left.\lambda \in \Lambda_{A}\right)$, or $\left(\chi=e\right.$ and $\left.\lambda \in \Lambda_{B}\right)$. In both cases, we have $\chi R^{t-1, t} a$, i.e. the axiom is satisfied. $I V)$ Suppose that $\omega=d$, which in turn implies $\chi=e$. Then either $\left(S\left(R_{M}\right)=\left\{R^{t}, R^{t+1}\right\}\right.$ and $\left.\lambda \in \Lambda_{A}\right)$ or $\left(S\left(R_{M}\right)=\left\{R^{t-1}, R^{t}\right\}\right.$ and $\left.\lambda \in \Lambda_{B}\right)$. In the first case, we have $e R^{t, t+1} d$. In the second case, we have $e R^{t-1, t} d$. The axiom is therefore satisfied in both cases, which ends the proof.

If $\Pi$ satisfies Pareto-efficiency and population-monotonicity, then $\Pi=\Pi_{\lambda}$ for some $\lambda \in \Lambda$.

Let $\Pi$ be a solution on $\mathcal{U}_{5}$ satisfying these two conditions. Throughout the proof, for all population $M$, let $\theta_{M}:=\Pi\left(R_{M}^{* 5}\right)$. 
Step 1: Choice of a candidate vector $\lambda \in \Lambda$.

Let $x:=\theta_{\{0, \ldots, 4\}}$. Let $t^{\prime}$ be the unique index in $\{0, \ldots, 4\}$ such that $x \in$ ]$\left.c_{t+2}, c_{t-2}\right]$. Let $a^{\prime}:=\theta_{\left\{t^{\prime}, t^{\prime}+1, t^{\prime}+2\right\}}, b^{\prime}:=\theta_{\left\{t^{\prime}-2, t^{\prime}-1, t^{\prime}\right\}}, d^{\prime}:=\theta_{\left\{t^{\prime}-1, \ldots, t^{\prime}+1\right\}}$. By Pareto-efficiency, we have $a^{\prime} \in\left[c_{t^{\prime}}, c_{t^{\prime}+2}\right], b^{\prime} \in\left[c_{t^{\prime}-2}, c_{t^{\prime}}\right], d^{\prime} \in\left[c_{t^{\prime}-1}, c_{t^{\prime}+1}\right]$. By population-monotonicity ${ }_{+}$, we have $a^{\prime} R^{t^{\prime}+2} x$ and $b^{\prime} R^{t^{\prime}-2} x$, which further implies $a^{\prime} \in\left[c_{t^{\prime}+1}, c_{t^{\prime}+2}\right]$ and $b^{\prime} \in\left[c_{t^{\prime}-2}, c_{t^{\prime}-1}\left[\right.\right.$. By population-monotonicity $y_{+}$, we have $d^{\prime} R^{t^{\prime}+1} x$, and $d^{\prime} R^{t^{\prime}-1} x$. To define the last location $e^{\prime}$, we distinguish two cases $A$ and $B$, which exhaust all possibilities, since $d^{\prime} \in\left[c_{t^{\prime}-1}, c_{t^{\prime}+1}\right]$. Case $A$ : If $d^{\prime} \in\left[c_{t^{\prime}-1}, c_{t^{\prime}}\right]$, let $e^{\prime}:=\theta_{\left\{t^{\prime}, t^{\prime}+1\right\}}$. Case B: If $d^{\prime} \in\left[c_{t^{\prime}}, c_{t^{\prime}+1}\right]$, let $e^{\prime}:=\theta_{\left\{t^{\prime}-1, t^{\prime}\right\}}$. If $d^{\prime}=c_{t^{\prime}}$, by Lemma 3 , these two definitions of $e^{\prime}$ coincide in $e^{\prime}=c_{t^{\prime}}$, thus $e^{\prime}$ is well defined.

In Case A, population-monotonicity + implies $d^{\prime} R^{t^{\prime}-1} b^{\prime}, e^{\prime} R^{t^{\prime}+1} a^{\prime}$, and $e^{\prime}$ $R^{t^{\prime}} d^{\prime}$. In Case $\mathrm{B}$, it implies $d^{\prime} R^{t^{\prime}+1} b^{\prime}, e^{\prime} R^{t^{\prime}-1} a^{\prime}$, and $e^{\prime} R^{t^{\prime}} d^{\prime}$. The vector $\lambda:=\left(x, a^{\prime}, b^{\prime}, d^{\prime}, e^{\prime}\right)$ satisfies all the conditions of either the set $\Lambda_{A}$ (in Case $A$ ) or the set $\Lambda_{B}$ (in Case $B$ ), except perhaps the condition $a^{\prime} \neq c_{t^{\prime}+1}$.

Let $t^{\prime \prime}$ be the unique index in $\{0, \ldots, 4\}$ such that $x \in\left[c_{t+2}, c_{t-2}[\right.$. Observe that either $t^{\prime \prime}=t^{\prime}$ or $t^{\prime \prime}=t^{\prime}+1$. Define $a^{\prime \prime}, b^{\prime \prime}, d^{\prime \prime}, e^{\prime \prime}$ and $\lambda^{\prime \prime}$, in a similar fashion as $a^{\prime}, b^{\prime}, d^{\prime}$ and $e^{\prime}$. Again, the vector $\lambda:=\left(x, a^{\prime}, b^{\prime}, d^{\prime}, e^{\prime}\right)$ satisfies all the conditions of either $\Lambda_{A}$ or $\Lambda_{B}$, except perhaps the condition $b^{\prime \prime} \neq c_{t^{\prime}-1}$. We now prove that either $a^{\prime} \neq c_{t^{\prime}+1}$ or $b^{\prime \prime} \neq c_{t^{\prime \prime}-1}$ holds, which will ensure that either $\lambda^{\prime}$ or $\lambda^{\prime \prime}$ is an element of $\Lambda$.

First, if the prime variables coincide with the double prime variables, we have $x \in] c_{t^{\prime}+2}, c_{t^{\prime}-2}\left[\right.$. This, $a^{\prime} R^{t^{\prime}+2} x$ and $b^{\prime} R^{t^{\prime}-2} x$, imply that $\left(a^{\prime} \neq c_{t^{\prime}+1}\right.$ and $\left.b^{\prime} \neq c_{t^{\prime}-1}\right)$. Suppose that they differ. By definition, we have $t^{\prime \prime}=t^{\prime}+1$, and $b^{\prime \prime}=d^{\prime}$. Suppose for example that $a^{\prime}=c_{t^{\prime}+1}$. Then $a^{\prime} R^{t^{\prime}+2} x$ implies $x=c_{t^{\prime}-2}$. Then $b^{\prime} R^{t^{\prime}-2} x$ implies $b^{\prime}=c_{t^{\prime}-2}$. Then $d^{\prime} R^{t^{\prime}-1} x$ and $d^{\prime} \in\left[c_{t^{\prime}-1}, c_{t^{\prime}+1}\right]$ implies $d^{\prime} \in\left[c_{t^{\prime}-1}, c_{t^{\prime}}\right]$. Therefore, Case A holds, i.e. $e^{\prime} \in\left[c_{t^{\prime}}, c_{t^{\prime}+1}\right]$. Then $e^{\prime} R^{t^{\prime}+1} a^{\prime}$ implies $e^{\prime}=a^{\prime}$. Then $e^{\prime} R^{t^{\prime}} d^{\prime}$ implies $d^{\prime}=c_{t^{\prime}-1}$. Therefore, $b^{\prime \prime}=c_{t^{\prime}-1}=c_{t^{\prime \prime}-2} \neq$ 
$c_{t^{\prime \prime}-1}$. Therefore, either $\lambda^{\prime}$ or $\lambda^{\prime \prime}$ is an element of $\Lambda$. Let $\lambda \in\left\{\lambda^{\prime}, \lambda^{\prime \prime}\right\} \cap \Lambda$.

Step 2: We have $\Pi=\Pi_{\lambda}$, with $\lambda \in \Lambda$ defined as in Step 1.

By Pareto-efficiency, $\Pi$ and $\Pi_{\lambda}$ obviously coincide on all unanimous profiles. Let $R_{M}$ be any non-unanimous profile in $\mathcal{U}_{5}$. Suppose first that $x \in E\left(R_{M}\right)$. By Lemma 3, we have $\Pi\left(R_{M}\right)=x=\Pi_{\lambda}\left(R_{M}\right)$. Suppose instead that $x \notin$ $E\left(R_{M}\right)$ and $b \in E\left(R_{M}\right)$. Since $b \in\left[c_{t-2}, c_{t-1}\left[\right.\right.$, then either $E\left(R_{M}\right)=\left[c_{t-2}, c_{t}\right]$ or $E\left(R_{M}\right)=\left[c_{t-2}, c_{t-1}\right]$. In both cases, $E\left(R_{M}\right) \subseteq E\left(R_{\{t-2, t-1, t\}}\right)$. Thus by Lemma 3, we have $\Pi\left(R_{M}\right)=b=\Pi_{\lambda}\left(R_{M}\right)$. Suppose instead that $x \notin E\left(R_{M}\right)$ and $a \in E\left(R_{M}\right)$. By a symmetric argument, we have $\Pi\left(R_{M}\right)=a=\Pi_{\lambda}\left(R_{M}\right)$. Suppose instead that $x, a, b \notin E\left(R_{M}\right)$, and $d \in E\left(R_{M}\right)$. Then either $E\left(R_{M}\right)=$ $\left[c_{t-1}, c_{t+1}\right], E\left(R_{M}\right)=\left[c_{t-1}, c_{t}\right]$ or $E\left(R_{M}\right)=\left[c_{t}, c_{t+1}\right]$. In all cases, $E\left(R_{M}\right) \subseteq$ $E\left(R_{\{t-1, t, t+1\}}\right)$. Thus by Lemma 3 , we have $\Pi\left(R_{M}\right)=d=\Pi_{\lambda}\left(R_{M}\right)$. Suppose instead that $x, a, b, d \notin E\left(R_{M}\right)$ and $e \in E\left(R_{M}\right)$. Suppose further that $\lambda \in \Lambda_{A}$. Then $E\left(R_{M}\right)=\left[c_{t}, c_{t+1}\right]=E\left(R_{\{t, t+1\}}\right)$. Then by Lemma 3, we have $\Pi\left(R_{M}\right)=\theta_{\{t, t+1\}}=e=\Pi_{\lambda}\left(R_{M}\right)$. Suppose instead that $\lambda \in \Lambda_{B}$. Then $E\left(R_{M}\right)=\left[c_{t-1}, c_{t}\right]=E\left(R_{\{t-1, t\}}\right)$. Then by Lemma 3 , we have $\Pi\left(R_{M}\right)=$ $\theta_{\{t-1, t\}}=e=\Pi_{\lambda}\left(R_{M}\right)$. In conclusion, we proved that $\Pi=\Pi_{\lambda}$.

Proof of (ii).$\Phi_{\lambda}$ is well defined. We already proved that $\succ_{\lambda}$ satisfies conditions (1) and (2), for all $\lambda \in \Lambda$, in particular for all $\lambda \in \Lambda_{\cap}$.

$\Phi_{\lambda}$ satisfies replacement-domination. Let $\lambda \in \Lambda_{\cap}, i \in N, L:=N \backslash\{i\}$, $R_{L} \in \mathcal{R}_{5}^{L}$ and $R_{i}, R_{i}^{\prime} \in \mathcal{R}_{5}$. Let $\omega:=\Phi_{\lambda}\left(R_{i}, R_{L}\right)$ and $\omega^{\prime}:=\Phi_{\lambda}\left(R_{i}^{\prime}, R_{L}\right)$. We will show that replacement-domination is satisfied, i.e. either $\omega R_{L} \omega^{\prime}$, or $\omega^{\prime} R_{L}$ $\omega$. Observe that we also have $\omega=\Pi_{\lambda}\left(R_{i}, R_{L}\right)$ and $\omega^{\prime}=\Pi_{\lambda}\left(R_{i}^{\prime}, R_{L}\right)$ and that $\Pi_{\lambda}$ satisfies population-monotonicity. Let $\chi:=\Pi_{\lambda}\left(R_{L}\right)$. First, by populationmonotonicity, we have $\chi R_{L} \omega$ and $\chi R_{L} \omega^{\prime}$. Therefore, if $\chi \in\left\{\omega, \omega^{\prime}\right\}$, the axiom is satisfied. Suppose through the end of the proof that $\chi \notin\left\{\omega, \omega^{\prime}\right\}$. Since $E\left(R_{L}\right) \subset E\left(R_{i}, R_{L}\right)$, we have $\omega \succ_{\lambda} \chi$. For similar reasons, we have $\omega^{\prime} \succ_{\lambda} \chi$. Second, if $\omega=\omega^{\prime}$, the axiom is trivially satisfied. Suppose through the 
end of the proof that $\omega \neq \omega^{\prime}$. Third, if $R_{L}$ is a unanimous profile, the axiom is trivially satisfied. Suppose through the end of the proof that this is not the case, which also implies that $\left(R_{i}, R_{L}\right)$ and $\left(R_{i}^{\prime}, R_{L}\right)$ are not unanimous either. To summarize, our suppositions are that $\omega, \omega^{\prime}$ and $\chi$ are distinct locations in $\left\{x, c_{t-2}, c_{t}, c_{t+2}\right\}$, such that $\omega \succ_{\lambda} \chi$ and $\omega^{\prime} \succ_{\lambda} \chi$. Since $\omega \neq \omega^{\prime}$, at least one of these two locations is distinct from $x$. Without loss of generality, suppose for example that $\omega \neq x$. Since we also have $\omega \succ \chi$, then $\omega \in\left\{c_{t-2}, c_{t+2}\right\}$. Without loss of generality, suppose for example that $\omega=c_{t+2}$. Then $\omega \neq \chi$ implies that $S\left(R_{L}\right)=\left\{R^{t}, R^{t+1}\right\}$ and $\chi=c_{t}$. This in turn implies that $\omega^{\prime} \neq c_{t-2}$. Since we also have $\omega^{\prime} \neq \omega, \chi$, then necessarily $\omega^{\prime}=x$. Since we have $c_{t+2} R^{t, t+1} x$, the axiom is satisfied, which ends the proof.

If $\Phi$ satisfies Pareto-efficiency and replacement-domination, then $\Phi=\Phi_{\lambda}$ for some $\lambda \in \Lambda_{\cap}$.

Let $\Phi$ be a solution on $\mathcal{R}_{5}^{N}$ satisfying these two conditions. By Theorem 2, $\Phi$ can be extended into a solution on $\mathcal{U}_{5}$ that satisfies population-monotonicity. Therefore, there exists $\lambda=(x, a, b, d, e) \in \Lambda$ such that $\Pi_{\lambda}$ coincides with $\Phi$, which in turn implies $\Phi=\Phi_{\lambda}$. We prove that $\lambda \in \Lambda_{\cap}$. Suppose by contradiction that $\lambda \notin \Lambda_{A}$. Consider $R_{N}:=\left(R^{t+1}, R^{t-1}, R^{t}, \ldots, R^{t}\right) \in \mathcal{R}_{5}^{N}$ and $R_{N}^{\prime}:=\left(R^{t-2}, R^{t-1}, R^{t}, \ldots, R^{t}\right) \in \mathcal{R}_{5}^{N}$. We have $\left.\left.\Phi\left(R_{N}\right)=d \in\right] c_{t}, c_{t+1}\right]$ and $\Phi\left(R_{N}^{\prime}\right)=b \in\left[c_{t-2}, c_{t-1}[\right.$. But this violates replacement-domination for preferences $R^{t-1}$ and $R^{t}$, a contradiction. Therefore, $\lambda \in \Lambda_{A}$. A similar reasoning proves $\lambda \in \Lambda_{B}$. In conclusion $\Phi=\Phi_{\lambda}$, for some $\lambda \in \Lambda_{\cap}$

\subsection{A negative result}

Unfortunately, the existence results obtained for polygons of order 3,4 and 5 do not extend to larger polygons. When $v \geq 6$, no solution on $\mathcal{U}_{v}$ satisfies Pareto-efficiency and population-monotonicity. A fortiori, no solution on $\mathcal{R}_{v}^{N}$ satisfies Pareto-efficiency and replacement-domination. 
Theorem 6 Let $v \geq 6$. No solution on $\mathcal{U}_{v}$ satisfies Pareto-efficiency and population monotonicity. No solution on $\mathcal{R}_{v}^{N}$ satisfies Pareto-efficiency and replacementdomination.

Proof. Let $\eta:=\lfloor v / 2\rfloor$. Since $v \geq 6$, then $\eta \geq 3$. Suppose, by contradiction, that $\Pi$ is a solution on $\mathcal{U}_{v}$ satisfying Pareto-efficiency and populationmonotonicity. For all population $M$, let $\theta_{M}:=\Pi\left(R_{M}^{* v}\right)$. Let $s$ be the unique integer such that $\theta_{\{0, \ldots, v-1\}} \in\left[c_{s}, c_{s+1}[\right.$. For simplicity and without loss of generality, assume that $s=0$, so that $\theta_{\{0, \ldots, v-1\}} \in\left[c_{0}, c_{1}[\right.$.

By population-monotonicity, , we have $\theta_{\{2, \ldots, 2+\eta\}} R^{2,2+\eta} \theta_{\{0, \ldots, v-1\}}$. Since $\theta_{\{0, \ldots, v-1\}} \in\left[c_{0}, c_{1}\left[\right.\right.$ and $\theta_{\{2, \ldots, 2+\eta\}} R^{2} \theta_{\{0, \ldots, v-1\}}$, therefore $\theta_{\{2, \ldots, 2+\eta\}} \in\left[c_{0}, c_{4}\right]$. Since $\theta_{\{0, \ldots, v-1\}} \in\left[c_{0}, c_{1}\left[\right.\right.$ and $\theta_{\{2, \ldots, 2+\eta\}} R^{2+\eta} \theta_{\{0, \ldots, v-1\}}$, then $\left.\theta_{\{2, \ldots, 2+\eta\}} \in\right] c_{2}, c_{1}[$. In addition, by Pareto-efficiency, $\theta_{\{2, \ldots, 2+\eta\}} \in\left[c_{2}, c_{2+\eta}\right]$. Thus, $\theta_{\{2, \ldots, 2+\eta\}} \in$ ]$\left.c_{2}, c_{4}\right]$.

Since $\{3,4,5\} \subseteq\{2, \ldots, 2+\eta\}$, then by population-monotonicity ${ }_{+}$, we have $\theta_{\{3,4,5\}} R^{3} \theta_{\{2, \ldots, 2+\eta\}}$. This relation and $\left.\left.\theta_{\{2, \ldots, 2+\eta\}} \in\right] c_{2}, c_{4}\right]$ imply $\theta_{\{3,4,5\}} \in\left[c_{2}, c_{4}\right]$. But by Pareto-efficiency, $\theta_{\{3,4,5\}} \in\left[c_{3}, c_{5}\right]$. Therefore, $\theta_{\{3,4,5\}} \in\left[c_{3}, c_{4}\right]$.

By population-monotonicity ${ }_{+}$, we have $\theta_{\{3, \ldots, 3+\eta\}} R^{3,3+\eta} \theta_{\{0, \ldots, v-1\}}$. Since $\theta_{\{0, \ldots, v-1\}} \in\left[c_{0}, c_{1}\left[\right.\right.$ and $\theta_{\{3, \ldots, 3+\eta\}} R^{3} \theta_{\{0, \ldots, v-1\}}$, therefore $\theta_{\{3, \ldots, 3+\eta\}} \in\left[c_{0}, c_{6}\right]$. Since $\theta_{\{0, \ldots, v-1\}} \in\left[c_{0}, c_{1}\left[\right.\right.$ and $\theta_{\{3, \ldots, 3+\eta\}} R^{3+\eta} \theta_{\{0, \ldots, v-1\}}$, then $\left.\theta_{\{3, \ldots, 3+\eta\}} \in\right] c_{4}, c_{1}[$. In addition, by Pareto-efficiency, $\theta_{\{3, \ldots, 3+\eta\}} \in\left[c_{3}, c_{3+\eta}\right]$. Thus, $\theta_{\{3, \ldots, 3+\eta\}} \in$ ]$\left.c_{4}, c_{6}\right]$.

By population-monotonicity + , we have $\theta_{\{3,4,5\}} R^{5} \theta_{\{3, \ldots, 3+\eta\}}$, and by Paretoefficiency, $\theta_{\{3,4,5\}} \in\left[c_{3}, c_{5}\right]$. Thus, $\theta_{\{3,4,5\}} \in\left[c_{4}, c_{5}\right]$. Since $\theta_{\{3,4,5\}} \in\left[c_{4}, c_{5}\right]$ and $\theta_{\{3,4,5\}} \in\left[c_{3}, c_{4}\right]$, we have $\theta_{\{3,4,5\}}=c_{4}$. Since $c_{4}=\theta_{\{3,4,5\}} R^{3} \theta_{\{2, \ldots, 2+\eta\}}$ and $\left.\left.\theta_{\{2, \ldots, 2+\eta\}} \in\right] c_{2}, c_{4}\right]$, we have $\theta_{\{2, \ldots, 2+\eta\}}=c_{4}$. By population-monotonicity, , we have $\theta_{\{2,3,4\}} R^{4} \theta_{\{2, \ldots, 2+\eta\}}=c_{4}$. Thus, $\theta_{\{2,3,4\}}=c_{4}$. By population-monotonicity ${ }_{+}$, we have $c_{4}=\theta_{\{2,3,4\}} R^{2} \theta_{\{1, \ldots, 4\}}$ and by Pareto-efficiency, $\theta_{\{1, \ldots, 4\}} \in\left[c_{1}, c_{4}\right]$. Thus, $\theta_{\{1, \ldots, 4\}}=c_{4}$. By population-monotonicity ${ }_{+}$, we have $c_{4}=\theta_{\{1, \ldots, 4\}} R^{1} \theta_{\{0, \ldots, v-1\}}$. 
But this result contradicts $\theta_{\{0, \ldots, v-1\}} \in\left[c_{0}, c_{1}\left[\right.\right.$. Therefore, no solution on $\mathcal{U}_{v}$ satisfies Pareto-efficiency and population-monotonicity. A fortiori by Theorem 2, no solution on $\mathcal{R}_{v}^{N}$ satisfies Pareto-efficiency and replacement-domination.

This result implies Theorem 1.

Proof of Theorem 1. The proof is by contradiction. Suppose that $\Pi$ (respectively $\Phi$ ) is a solution on $\mathcal{U}$ (respectively on $\mathcal{R}^{N}$ ) satisfying Pareto-efficiency and population-monotonicity (respectively replacement-domination). Consider the restriction $\Pi^{\prime}$ of $\Pi$ (respectively $\Phi^{\prime}$ of $\left.\Phi\right)$ to $\mathcal{U}_{6}$ (respectively to $\mathcal{R}_{6}^{N}$ ). Then $\Pi^{\prime}$ (respectively $\Phi^{\prime}$ ) satisfies Pareto-efficiency and population-monotonicity (respectively replacement-domination), in contradiction with Theorem 6.

All results on population-monotonicity in the paper were established under the assumption that $\mathcal{N}$ is countably infinite. This assumption is convenient in the proofs, but not essential. As long as $\mathcal{N}$ contains at least four agents, the results on population-monotonicity are not affected. Proving this requires intermediate results on population-monotonicity in the spirit of Lemma 4 and Theorem 2, and long but not difficult proofs. All results on replacement-domination in the paper were established for a fixed population containing at least four agents. For both problems, the case of three agents is an open question.

\section{Conclusion}

We have shown that Pareto-efficiency and solidarity are incompatible in sufficiently rich cyclically single-peaked domains, and have characterized the solutions satisfying these properties for smaller polygon-structured domains. The solutions we characterized have the interesting property that each of them maximizes a certain weak partial order on the Pareto-set. Interestingly, the solutions we obtained also satisfy various appealing conditions, which we did not 
require. The solutions are all strategy-proof, and even coalition-strategy-proof, i.e. no coalition of agents can obtain a strict Pareto-improvement for all of its members by misreporting their preferences. The solutions are also essentially anonymous, i.e. each of them does not depend on agents' labels up to Paretoindifference ${ }^{10}$. An interesting question concerns solutions satisfying Paretoefficiency, anonymity, and strategy-proofness (or coalition-strategy-proofness) in smaller polygon-structured domains. In particular, how small does such a domain need to be, for these conditions to be compatible? Our results shows that the threshold polygon order for this problem is equal or larger than 5 . How large are the corresponding sets of solutions?

\section{References}

[1] Barberà, Salvador and Matthew O. Jackson, A Characterization of Strategy-proof Social Choice Functions for Economies with Pure Public Goods, Social Choice and Welfare 11, 241-252, 1994.

[2] Ching, Steven and William Thomson, Population-Monotonicity in Public Good Economies with Single-Peaked Preferences, Working Paper, University of Rochester, forthcoming in Social Choice and Welfare 1999.

[3] Ehlers, Lars, Multiple Public Goods and Lexicographic Preferences: Replacement Principle, Journal of Mathematical Economics, 37, 1-15, 2002.

[4] Ehlers, Lars, Multiple Public Goods, Lexicographic Preferences, and Single-Plateaued Preference Rules, Games and Economic Behavior, 43, 127, 2003.

\footnotetext{
${ }^{10}$ Gordon (2005) establishes general relations among these properties in a model of public choice, that contains location models as a special case.
} 
[5] Ehlers, Lars and Klaus, Bettina, Solidarity and Probabilistic Target Rules, Journal of Public Economic Theory, Vol. 3-2, 167-184, April 2001.

[6] Gordon, Sidartha, Public Decisions: Solidarity and the Status Quo, Working Paper, Université de Montréal, 2006.

[7] Gordon, Sidartha, Solidarity and Unanimity in Attribute-Based Domains, Working Paper, Université de Montréal, 2003.

[8] Klaus, Bettina, Target Rules for Public Choice Economies on Tree Networks and in Euclidean Spaces, Theory and Decision, 51 (1): 13-29, 2001.

[9] Miyagawa, Eiichi, Mechanisms for Providing a Menu of Public Goods: Population-Monotonicity, Working Paper, Columbia University, 1998.

[10] Miyagawa, Eiichi, Locating Two Libraries on a Street: Replacement Principle, Social Choice and Welfare, Vol 18, 527-541, 2001.

[11] Moulin, Herve, The Pure Compensation Problem: Egalitarianism versus Laissez-Fairism, Quarterly Journal of Economics, 102, 769-783, 1987.

[12] Moulin, Herve, On Strategy-Proofness and Single-Peakedness, Public Choice 35, 437-455, 1980.

[13] Schummer, James P. and Vohra, Rakesh V., Strategy-proof Location on a Network, Journal of Economic Theory, 104, 405-428, 2002.

[14] Thomson, William, The Fair Division of a Fixed Supply Among a Growing Population, Mathematics of Operations Research, Vol. 8, pp. 319-326, $1983 \mathrm{a}$.

[15] Thomson, William, Problems of Fair Division and the Egalitarian Solution, Journal of Economic Theory, 31, pp. 211-226, $1983 \mathrm{~b}$. 
[16] Thomson, William, The Replacement Principle in Public Good Economies with Single-Peaked Preferences, Economic Letters 42, pp. 31-36, 1993.

[17] Thomson, William, Population-Monotonic Allocation Rules. In: Barrett W., Moulin H., Sallles M., Schofield N. (eds.), Social Choice, Welfare, and Ethics. Cambridge University Press, Cambridge, pp. 79-124, 1995.

[18] Thomson, William, Welfare-domination under Preference-Replacement: a Survey and Open Questions, Social Choice and Welfare, 16, 373-394, 1999a.

[19] Thomson, William, Population-Monotonicity: a Survey and Open Questions, Working Paper, University of Rochester, 1999b.

[20] Vohra, Rakesh V., The Replacement principle and Tree Structured Preferences, Economic Letters, vol. 63, 175-180, 1999. 